\title{
Rapamycin reverses hypertrophic cardiomyopathy in a mouse model of LEOPARD syndrome-associated PTPN11 mutation
}

Talita M. Marin,1,2 Kimberly Keith, ${ }^{1}$ Benjamin Davies, ${ }^{1}$ David A. Conner, ${ }^{3}$ Prajna Guha, ${ }^{1}$ Demetrios Kalaitzidis, ${ }^{4}$ Xue Wu, ${ }^{5,6}$ Jessica Lauriol, ${ }^{1}$ Bo Wang, ${ }^{1}$ Michael Bauer, ${ }^{7}$ Roderick Bronson, ${ }^{8}$ Kleber G. Franchini, ${ }^{2}$ Benjamin G. Neel, ${ }^{, 6}$ and Maria I. Kontaridis ${ }^{1,9}$

1Department of Medicine, Division of Cardiology, Beth Israel Deaconess Medical Center, Boston, Massachusetts, USA. ${ }^{2}$ Department of Internal Medicine, School of Medicine, State University of Campinas, Campinas, Brazil. ${ }^{3}$ Department of Genetics and Howard Hughes Medical Institute, Harvard Medical School, Boston, Massachusetts, USA. ${ }^{4}$ Department of Hematology/Oncology, Children's Hospital, Boston, Massachusetts, USA.

5Department of Medical Biophysics, University of Toronto, Toronto, Ontario, Canada. ${ }^{6}$ Campbell Family Cancer Research Institute, Ontario Cancer Institute and Princess Margaret Hospital, University Health Network, Toronto, Ontario, Canada. ${ }^{7}$ Department of Medicine, Cardiovascular Division, Brigham and Women's Hospital, Boston, Massachusetts, USA. ${ }^{8}$ Department of Pathology and 9Department of Biological Chemistry and Molecular Pharmacology, Harvard Medical School, Boston, Massachusetts, USA

LEOPARD syndrome (LS) is an autosomal dominant "RASopathy" that manifests with congenital heart disease. Nearly all cases of LS are caused by catalytically inactivating mutations in the protein tyrosine phosphatase (PTP), non-receptor type 11 (PTPN11) gene that encodes the SH2 domain-containing PTP-2 (SHP2). RASopathies typically affect components of the RAS/MAPK pathway, yet it remains unclear how PTPN11 mutations alter cellular signaling to produce LS phenotypes. We therefore generated knockin mice harboring the Ptpn11 mutation Y279C, one of the most common LS alleles. Ptpn11 ${ }^{\mathrm{Y} 279 \mathrm{C} /+}(\mathrm{LS} /+)$ mice recapitulated the human disorder, with short stature, craniofacial dysmorphia, and morphologic, histologic, echocardiographic, and molecular evidence of hypertrophic cardiomyopathy (HCM). Heart and/or cardiomyocyte lysates from $L S /+$ mice showed enhanced binding of Shp2 to Irs1, decreased Shp2 catalytic activity, and abrogated agonist-evoked Erk/Mapk signaling. $L S /+$ mice also exhibited increased basal and agonist-induced Akt and $m$ Tor activity. The cardiac defects in $L S /+$ mice were completely reversed by treatment with rapamycin, an inhibitor of mTOR. Our results demonstrate that LS mutations have dominant-negative effects in vivo, identify enhanced mTOR activity as critical for causing LS-associated HCM, and suggest that TOR inhibitors be considered for treatment of HCM in LS patients.

\section{Introduction}

With approximately 1 per 100 babies affected each year, congenital heart diseases (CHDs) are the most common type of birth defect and the number 1 cause of birth-related deaths (1). Though numerous CHD phenotypes of various etiologies exist, the majority of patients present with abnormal valves, membranous septa, and/or myocardial defects (2). Abnormalities in signaling molecules and/or pathways involved in cardiac development are implicated in CHD pathogenesis; however, the underlying mechanisms remain poorly understood and/or unknown.

Primary hypertrophic cardiomyopathy (HCM), which affects the cardiac muscle wall, is a common cause of mortality and morbidity in CHD patients (3-5). Although hypertrophy may initially be a compensatory response to cardiac stress, over time, HCM can decompensate to dilation and end-stage heart failure. Mutations in genes that encode sarcomeric proteins, such as the myosin-bind-

Authorship note: Talita M. Marin and Kimberly Keith contributed equally to this work.

Conflict of interest: Maria I. Kontaridis and Benjamin G. Neel have filed a provisional patent application for the use of rapamycin and rapamycin analogs for the treatment of LEOPARD syndrome and related disorders.

Citation for this article: J Clin Invest. 2011;121(3):1026-1043. doi:10.1172/JCI44972. ing protein $\mathrm{C}, \beta-M H C$, and troponin $\mathrm{T}$, and that alter sarcomeric structure and function constitute approximately $75 \%$ of cases of primary HCM (6-9). The remaining approximately $25 \%$ are caused by mutations in other genes, most of which remain undefined. The ultimate effect of all of these mutations is to impose mechani$\mathrm{cal}$, biochemical and/or bioenergetic stresses on the heart. These stresses activate cardiac signaling pathways, including the RAS/ MAPK pathway, other MAPK (e.g., p38, JUN N terminal kinase [JNK]) pathways, the PI3K/AKT pathway, and in some cases the RHOA, STAT3, and/or calcineurin/nuclear factor of activated $\mathrm{T}$ cells pathways, to mediate the hypertrophic phenotype (10).

One non-sarcomeric protein involved in the pathogenesis of CHDs is SH2 domain-containing PTP-2 (SHP2), which is encoded by the non-receptor protein tyrosine phosphatase (PTP) type 11 (PTPN11) gene. SHP2 is a ubiquitously expressed protein that contains $2 \mathrm{~N}$ terminal SH2 domains, a catalytic (PTP) domain, and a $\mathrm{C}$ terminal tail with 2 tyrosine phosphorylation sites and a proline-rich motif $(11,12)$. SHP2 is regulated by an elegant molecular switch mechanism that couples its recruitment to appropriate locations in the cell with its catalytic activation $(13,14)$. In the basal state, the backside loop of the N-SH2 of SHP2 is wedged into the PTP domain, rendering the PTP domain physically and chemically inactive. Binding of a phosphotyrosyl 
peptide alters the conformation of the N-SH2 domain, preventing interaction of the backside loop with the PTP domain, unfolding the enzyme, and resulting in phosphatase activation (13-15).

Essential for development and the first PTP known to have a functional role in adult myocardium $(16,17)$, SHP2 plays a key role in RAS/MAPK activation in response to most, if not all, receptor tyrosine kinase (RTK), cytokine receptor, GPCR, and integrin signaling pathways (18). Moreover, germline PTPN11 mutations cause 2 related, but distinguishable, syndromes that include congenital heart defects among their features. Essentially all cases of LEOPARD syndrome (LS) (MIM151100), a rare autosomal dominant, multisystemic disease, are caused by mutations in PTPN11 (19). LS is named for its presenting manifestations: multiple lentigines, electrocardiographic conduction abnormalities, occular hypertelorism, pulmonic stenosis, abnormal genitalia, retardation of growth, and sensorineural deafness (20). PTPN11 mutations also cause more than $45 \%$ of cases of Noonan syndrome (NS) (MIM163950) (21), the more common disorder $(\sim 1 / 2,000$ births), with the remaining cases resulting from mutations in SOS1 (22, 23), KRAS (24), NRAS (25), SHOC2 (26), RAF1 (27, 28), or as yet unknown genes.

NS and LS are 2 of several autosomal dominant "RASopathies," disorders caused by mutations in various RAS/MAPK pathway components $(29,30)$. Not surprisingly, many of the phenotypic abnormalities in NS and LS (and in other RASopathies) are quite similar: for example, both are characterized by proportionate short stature, facial dysmorphism, and skeletal anomalies (21, 31). However, their cardiac phenotypes are quite distinct. Pulmonary valve stenosis with dysplastic leaflets and atrial/ventricular septal defects are the most prevalent cardiac defects in NS caused by PTPN11 mutations $(32,33)$. By contrast, despite the fact that pulmonic stenosis is part of the LS acronym, the most common cardiac manifestation of this disorder is HCM (34-38).

We and others previously showed that the biochemical properties of LS and NS alleles also are quite distinct (39-41), suggesting different mechanisms for NS and LS pathogenesis. Whereas NS mutants primarily perturb the N-SH2/PTP interface to preferentially "open" the structure to an activated state (39), LS mutations typically affect conserved residues important for PTP catalysis (e.g., of the 2 most common sites for mutation in LS, Y279 sets the depth of the catalytic cleft and T468 lies close to the PTP domain "signature motif"). Almost all NS mutants tested behave as gain-of-function mutations of SHP2 and act primarily, if not exclusively, to potentiate RAS/MAPK pathway activation (40, 42-46). By contrast, LS mutants are catalytically impaired and act as dominant-negative mutants, impairing ERK activation in transient and stable transfection assays (39-41).

Together, these findings raise an interesting question: how can PTPN11 mutations that result in SHP2 proteins with opposite catalytic functions give rise to such similar disease phenotypes? Drosophila (47) and Zebrafish $(48,49)$ models of LS have provided some insights; however, these data are conflicting. Oishi et al. reported that LS mutants enhance Ras/Mapk pathway activity in Drosophila (47), whereas in Zebrafish studies, Jopling et al. and Stewart et al. found that LS mutants have dominant-negative effects on this pathway $(48,49)$. To resolve this paradox and to address the above question in a potentially more relevant mammalian system, we generated an inducible "knockin" mouse model expressing the LS-associated Ptpn11 Y279C mutation (Ptpn11 1279 C/+ mice). Remarkably, our mice recapitulated nearly all major aspects of the LS phenotype. Furthermore, biochemical analyses of hearts and primary cardiomyocytes from these mice identify the molecular basis for HCM in LS and a pharmacological intervention that reverses the cardiac defects associated with LS in vivo.

\section{Results}

Generation of mice expressing an inducible Ptpn11 Y279C allele. To assess the functional consequences of LS mutations, we generated knockin mice harboring an inducible, floxed Y279C allele of Ptpn11, Ptpn $11^{\mathrm{Y279C/+}}$ mice, hereafter termed iLS/+ mice (Figure 1). We chose this allele because PTPN11 $1^{\mathrm{Y} 279 \mathrm{C}}$ is one of the 2 most common LS mutations $(40,50)$. The targeting construct was generated using recombination-mediated genetic engineering (recombineering) (51). Briefly, a fragment of the mouse Ptpn11 gene containing exons 7-9 was subcloned from a bacterial artificial chromosome clone into a plasmid containing a thymidine kinase (TK) selection cassette (for negative selection). The targeting vector itself included a loxP site, a Ptpn11 genomic fragment encoding WT exon 7, a loxP511 site, an inverted mutant Ptpn11 exon 7 fragment containing the Y279C mutation, an inverted loxP site, an inverted loxP511 site, a neo selection cassette flanked by FRT sites, and the Ptpn11 genomic fragment encoding WT exons 8 and 9 (Figure 1A). The inverted mutant Y279C exon 7 also introduced $2 \mathrm{HpyCH} 4$ restriction sites which we believe to be novel in the sequence to be used for screening (Figure 1). This construct, when recombined appropriately into the endogenous Ptpn11 locus, directs expression of WT Ptpn11 in the absence of Cre recombinase (Cre); these mice are termed $\mathrm{L} L \mathrm{~S} /+$ mice. Upon exposure to $\mathrm{Cre}$, unidirectional recombination (as a consequence of the mutant loxP sites) results in expression of the mutant $\mathrm{Y} 279 \mathrm{C}$ allele; these mice are designated $L S /+$ mice (Figure 1, A-C).

The targeting construct was electroporated into TC1 (129S6/ SvEv) ES cells and selected with G418 and FIAU. Resistant colonies were expanded, and homologous recombinants were identified by long-range PCR (Figure 1D and Supplemental Table 1; supplemental material available online with this article; doi:10.1172/ JCI44972DS1). Correctly targeted ES cell clones were injected into blastocysts and implanted into pseudopregnant mice. Germline transmission was achieved, as verified by PCR for the neo gene (Figure 1E and Supplemental Table 1). The neo cassette was then removed by crossing $i L S /+$ chimeras to Flp-recombinase-expressing mice (Figure 1B), and ubiquitous expression of the LS mutant was achieved by mating appropriate progeny from this cross with EIIA-Cre (C57BL6/J) mice (Figure 1C). These mice were subsequently crossed to WT C57BL6/J mice to generate mice with global LS expression $(L S /+$ mice). Two PCR strategies were used to distinguish $L S /+$ mice from WT mice using tail DNA (Figure 1F and Supplemental Table 1). Moreover, expression of the mutant allele (at levels comparable to that of WT Ptpn11 mRNA) was detected by RT-PCR, and the expected products were observed upon $\mathrm{HpyCH} 4$ digestion (Figure 1G and Supplemental Table 1). LS/+ mice also expressed comparable levels of Shp2 protein as compared to those of WT mice (data not shown). LS/+ mice were obtained at the expected Mendelian ratio at weaning, indicating that on this background, LS expression was compatible with embryonic development. All experiments herein were performed using $L S /+$ mice (and their WT littermates) crossed for at least 4-5 generations on C57BL6/J background.

$L S /+$ mice demonstrate phenotypic abnormalities similar to those in buman LS. It was immediately apparent that, compared with WT littermates, $L S /+$ mice had abnormal faces, with distinctly smaller, more slanted eyes (Figure 2A, top) and a planar nasal bridge 
A Targeting locus

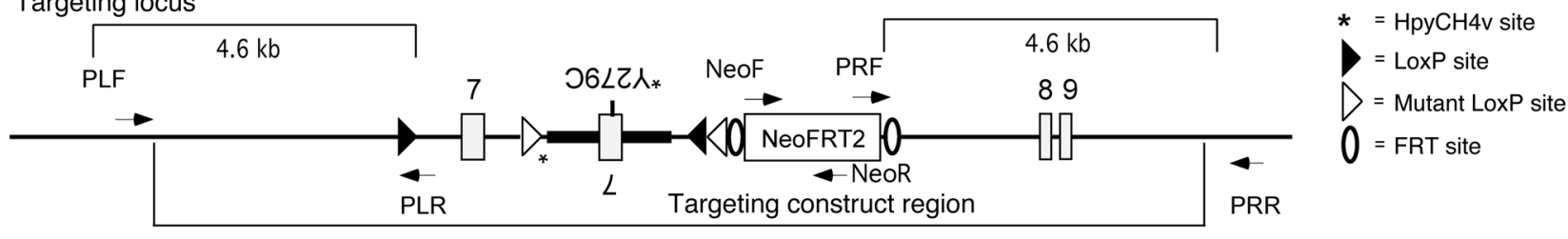

$\downarrow$ Flp recombinase

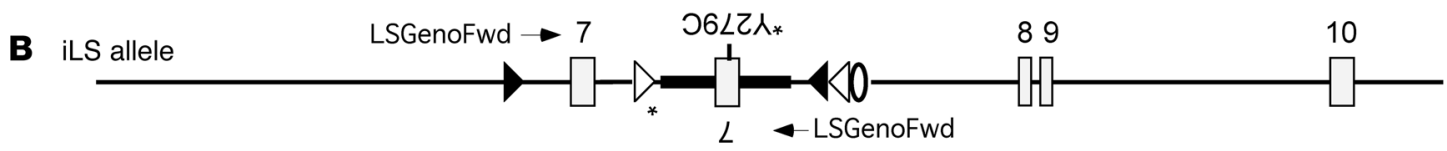

$\downarrow$ Cre recombinase inversion

C LS allele

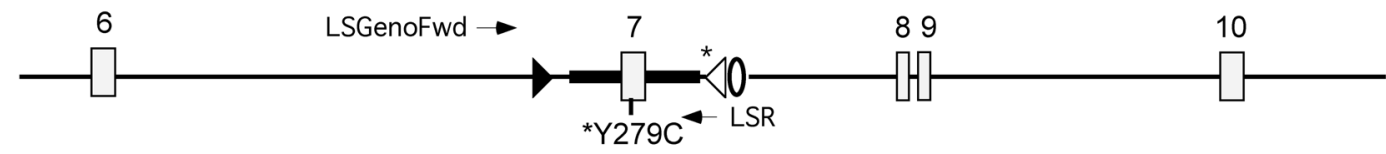

D

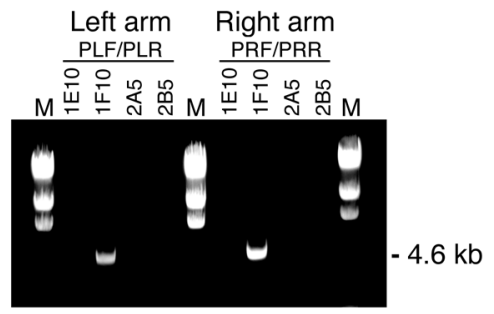

$\mathbf{E}$

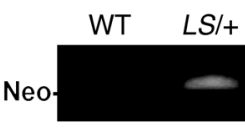

Neo Fwd/Rev
F

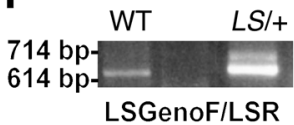

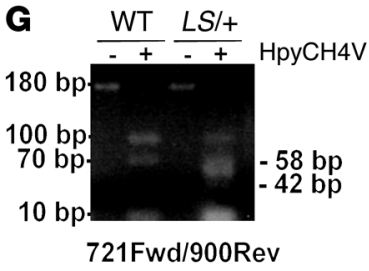

Figure 1

Generation of mice expressing an inducible Ptpn11 Y279C allele. (A) Structure of the Ptpn11 targeting locus and targeting construct region. Protein coding regions, LoxP sites, and Frt sites are shown. The arrows highlight the target positions of PCR primers used to identify homologous recombinants. Note that primers PLF and PRR are outside the region of homology. The expected amplicons are indicated with brackets and are specific to homologously recombined templates. LoxP sites are shown as black triangles; mutant LoxP511 sites are shown as white triangles. Their relative orientation is shown by the orientation of the triangles. FRT sites are shown as white ovals. Exons are indicated by rectangles. (B) Structure of locus after Flp-mediated recombination. (C) Structure of locus after Cre-mediated recombination. (D) Confirmation of correct targeting of ES cell clone $1 \mathrm{~F} 10$ by PCR, revealing a $4.6-\mathrm{kb}$ fragment on each correctly targeted side of the allele. M indicates molecular weight markers. (E) Germline transmission in F1 mice, as depicted by PCR, for the presence of the neo gene. (F) PCR detection strategy using LSGenofwd and LSR primers for detection of WT and $L S /+$ mice. (G) Restriction digest (HpyCH4V) of Ptpn11 exon 7 RT-PCR-derived fragment confirming expression of the mutant allele (full-length = $185 \mathrm{bp}$; fragment sizes = 100, $75 \mathrm{bp}, 58 \mathrm{bp}, 42 \mathrm{bp}$, and $10 \mathrm{bp}$ [LS]; $100 \mathrm{bp}, 75 \mathrm{bp}$, and $10 \mathrm{bp}$ [WT]). Note that the 10-bp fragment probably comigrates with primer alone and/or contaminating RNA. PCR primers used are 721 forward/900 reverse. See Supplemental Table 1 for details on primers used throughout this figure. Fwd, forward; Rev, reverse.

(Figure 2A, bottom). Microcomputed tomography ( $\mu \mathrm{CT}$ ) scans showed an increased skull width-to-length ratio in $L S /+$ mice, compared with that in WT controls (Table 1). Together, these features gave $L S /+$ mice the appearance of hypertelorism, even though inner canthal distance, while trending wider in $L S /+$ mice, was not statistically different. $L S /+$ mice also had skeletal/chest abnormalities, with pectus carinatum superiorly and pectus excavatum inferiorly (Figure 2B), a common feature of LS patients. Although their size was normal at birth (data not shown), by weaning, $L S /+$ mice were significantly smaller than their WT littermates, and they remained so throughout their lives (Figure 2, $C$ and D). $\mu$ CT scans showed that femur length also was decreased significantly in $L S /+$ mice (Table 1 ). Histological examination revealed abnormal genitalia and substantial macrophage accumulation in the organ of Corti (inner ear) in the LS/+ mice, which might cause (or indicate) sensorineural defects (data not shown). No other phenotypic/organ abnormalities were observed.
Somatic mutations in PTPN11 occur in several types of hematologic malignancies, most notably, juvenile myelomonocytic leukemia, and, more rarely, in solid tumors. Furthermore, approximately $20 \%-50 \%$ of NS patients have some hematologic abnormality (52-54). We collected peripheral blood, bone marrow, spleen, and thymus from a small cohort of either WT or LS/+ mice. Notably, in contrast to mice with NS-associated Ptpn11 mutations (43), we observed no apparent abnormalities in any hematological parameter in $L S /+$ mice (data not shown). Indeed, LS patients also are not reported to have any highly penetrant hematological abnormalities. Taken together, these data indicate that the gross morphologic features in $L S /+$ mice closely recapitulate those in LS patients $(20,31,55)$.

$L S /+$ mice have pathological cardiac bypertrophy that transitions to dilation. Over $80 \%$ of LS patients develop severe HCM $(38,55-57)$. Therefore, we focused on the cardiac effects of the Y279C mutation in our mice. Although normally sized at birth, $L S /+$ hearts became 

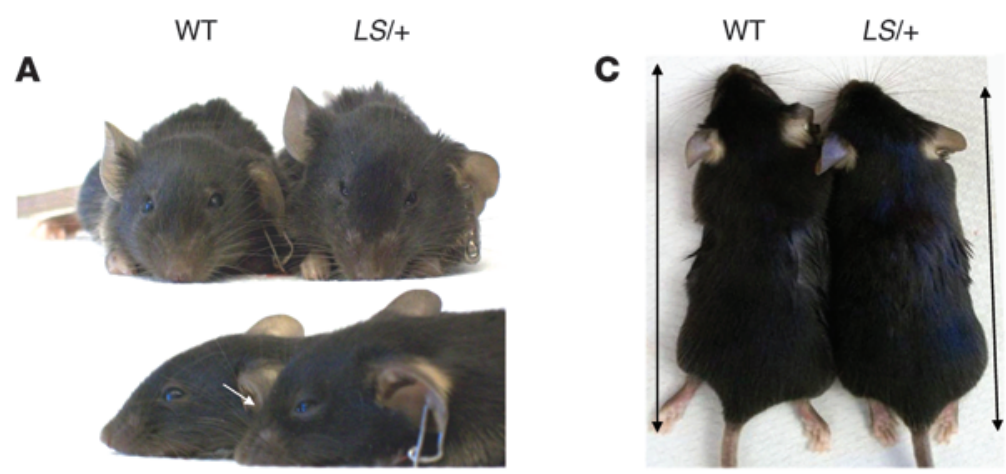

B

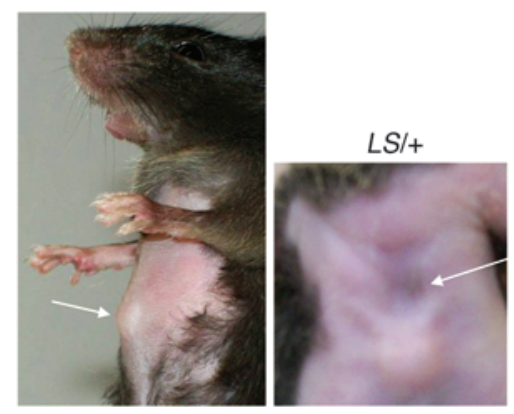

Figure 2
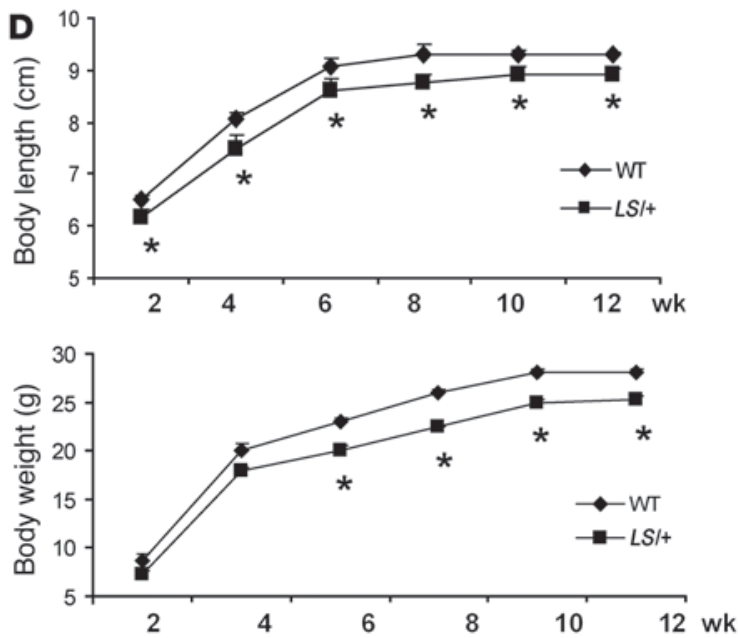

LS/+ mice demonstrate phenotypic abnormalities similar to those in human LS. (A) Representative photographs of en face and nose bridge profile (arrow) (image was brightened to enable a clear view of the facial phenotype). (B) Skeletal/chest deformity in $L S /+$ mice, with pectus carinatum superiorly (arrow) and pectus excavatum inferiorly (arrow). (C) Overall smaller body size in LS/+ mice as compared with that of WT mice. (D) Body length and weight are significantly less in $L S /+$ mice than in WT mice; $n=5-10$ mice/group at each age measured. Data represent mean \pm SEM; ${ }^{*} P<0.05$, 2-tailed Student's $t$ test.

progressively larger than WT hearts, becoming statistically different by 12 weeks of age (Figure 3, A and B, and data not shown). Analysis of H\&E-stained transverse sections of 16-week-old hearts revealed dramatic thickening of the left ventricular free wall and septum (Figure 3B). Consistent with these observations, heart weight to body weight ratios were significantly elevated in hearts from 12- and 16-week-old LS/+ mice (Figure 3C). Morphometric analysis of individual cardiomyocytes in reticulin-stained heart sections from 16-week-old mice (Figure 3D), or that of isolated primary adult cardiomyocytes from 8- to 10 -week-old $L S /+$ or WT mice (Figure 3E), indicated that the LS mutation did not affect overall cardiomyocyte number but rather significantly increased individual cardiomyocyte area (by $\sim 30 \%$ ), as compared with that of controls. Isolated cardiomyocytes also had significantly increased cell width as opposed to length (Figure 3E), consistent with concentric HCM (58).

Further indicative of pathological hypertrophy in LS/+ hearts, we observed markedly enlarged nuclei, myofiber disarray, and inflammatory cell accumulation in the interstitial spaces between myofibers of $L S /+$ hearts (Figure 3F, indicated by arrows and magnified in inset). Electron microscopy of 12 -week-old $L S /+$ hearts confirmed the increase in myofibril size but showed no overt effects on sarcomeric structure or mitochondrial number or size (Figure 3G).

\section{Table 1}

$\mu \mathrm{CT}$ morphometric values from WT and $L S /+$ mice

$\begin{array}{lcccc}\text { Genotype } & \text { Femur length }(\mathbf{m m}) & \text { Tibia length }(\mathbf{m m}) & \text { Skull width/skull length }(\mathbf{m m}) & \text { Inner canthal distance/skull width }(\mathbf{m m}) \\ \text { WT mice } & 15.66 \pm 0.16 & 17.19 \pm 0.14 & 0.447 \pm 0.002 & 0.635 \pm 0.003 \\ \text { LS/+ mice } & 15.07 \pm 0.14^{\mathrm{A}} & 17.02 \pm 0.24 & 0.457 \pm 0.004^{\mathrm{A}} & 0.642 \pm 0.010\end{array}$



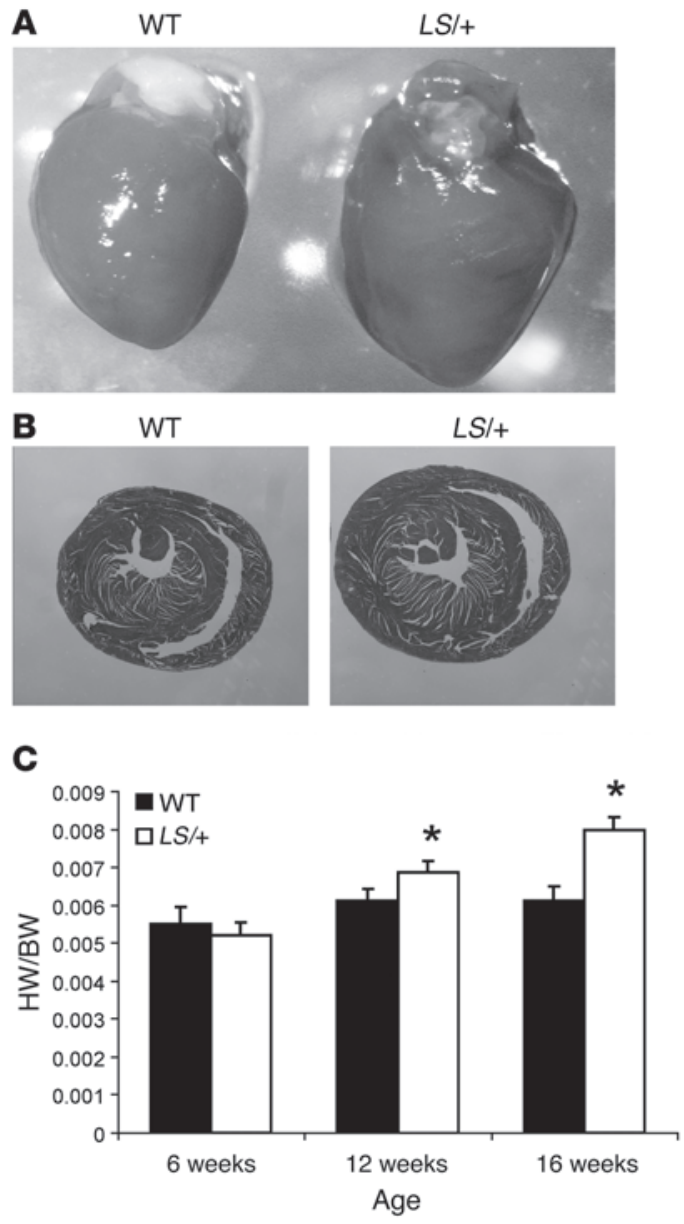
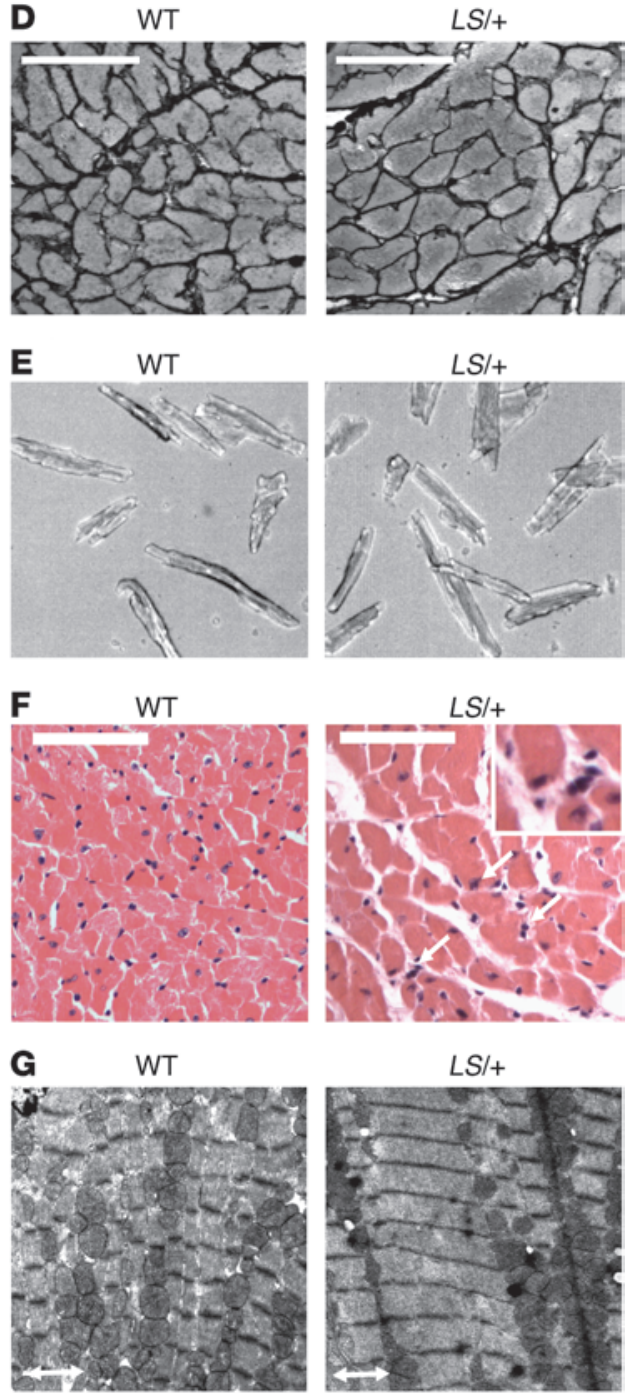

\section{Figure 3}

LS/+ mice have pathological cardiac hypertrophy. (A) Representative hearts from 16-week-old WT and $L S /+$ mice. (B) Transverse sections of WT and $L S /+$ hearts stained with H\&E (original magnification, $\times 100)$. (C) Heart weight to body weight $(H W / B W)$ ratios of WT and $L S /+$ mice at indicated ages $\left(n=5-10 \mathrm{mice} /\right.$ group at each age measured). Data represent mean $\pm \mathrm{SEM}$; ${ }^{\star} P<0.05,2$-tailed Student's $t$ test. (D) Paraffin-embedded heart sections from 16-week-old WT and $L S /+$ mice stained with reticulin (original magnification, $\times 400$; scale bar: $100 \mu \mathrm{m}$ ). The average area of WT cardiomyocytes is 2,038 $\pm 98.5 \mu \mathrm{m}^{2} /$ cardiomyocyte and that of $L S /+$ cardiomyocytes is $2,578 \pm 115.9 \mu \mathrm{m}^{2} /$ cardiomyocyte $(P<0.001$, 2-tailed Student's $t$ test). Data represent mean \pm SEM (200-500 cardiomyocytes/group). (E) Cardiomyocytes from 8- to 10-week-old WT and $L S /+$ hearts (original magnification, $\times 200$ ). Cardiomyocyte length for WT hearts is $108.4 \pm 2.6 \mu \mathrm{m}$ and for $L S /+$ hearts is $113.5 \pm 2.9 \mu \mathrm{m}$; cardiomyocyte width for WT hearts is $18.9 \pm 0.8 \mu \mathrm{m}$ and for $L S /+$ hearts is $22.4 \pm 0.7 \mu \mathrm{m}(P<0.05,2$-tailed Student's $t$ test). Data represent mean \pm SEM (200-500 cardiomyocytes; $n=4$ mice each.). (F) H\&E staining (original magnification, $\times 400$; white bar: $100 \mu \mathrm{m}$ ) of paraffin-embedded longitudinal heart sections from 16-week-old WT and LS/+ mice. Note the myofiber disarray and increased inflammatory cells (indicated by white arrows and magnified in inset) in the interstitial spaces. (G) Scanning electron microscopy of cardiomyocytes (original magnification, $\times 4,800$ ) from 12-week-old mice. Double arrowhead: $1 \mu \mathrm{m}$.

By 52 weeks, we noted a significantly increased number of deaths in the $L S /+$ mouse cohort (Figure 4A). Hearts from the older $L S /+$ mice displayed increased myofiber disarray, enlarged nuclei (Figure 4B), and marked fibrosis (Figure 4C), yet their cardiomyocytes remained larger than those of WT (Figure 4D). Although the precise reason(s) of excess mortality in $L S /+$ mice cannot be ascertained with certainty, these pathologic features strongly implicate cardiac causes (e.g., end-stage heart failure or arrhythmia).
To assess cardiac function and morphology more quantitatively, we obtained echocardiographs of $L S /+$ and WT littermates at $6,12,16,20$, and 52 weeks (Figure 5 and Supplemental Figure 1). No differences in cardiac size or function were observed at 4 to 6 weeks of age. By 12 weeks, however, LS/+ mice had significant left ventricular hypertrophy, as indicated by decreased chamber dimension and increased posterior wall thickness compared with those of littermate controls. Furthermore, $L S /+$ mice did indeed transition to dilated cardiomyopathy by 52 weeks, with 
A
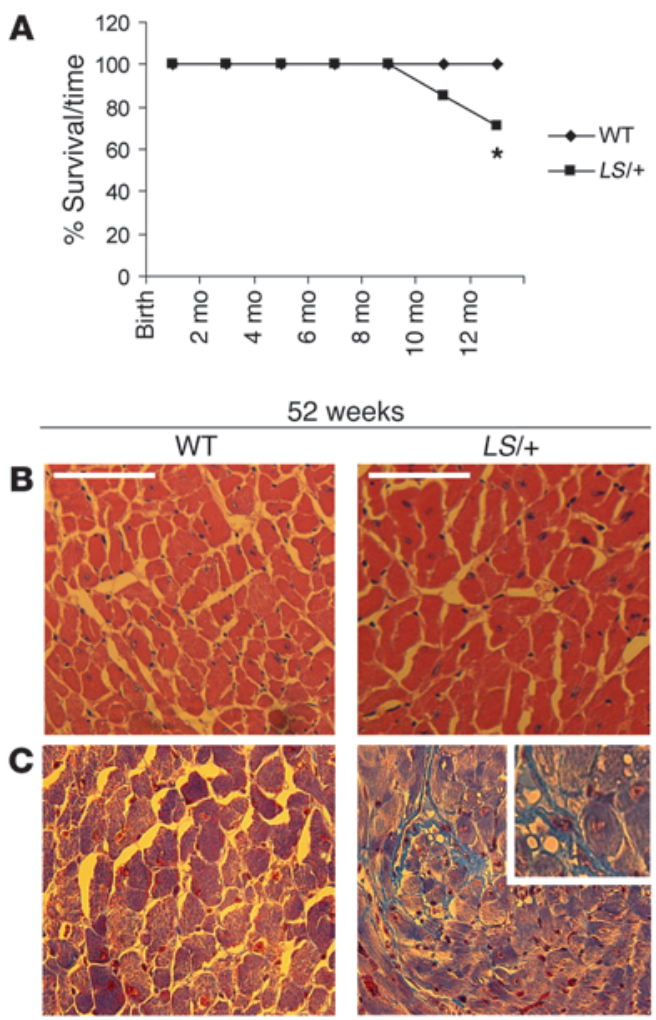

D

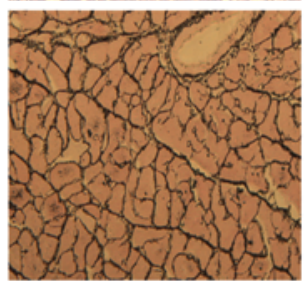

52 weeks
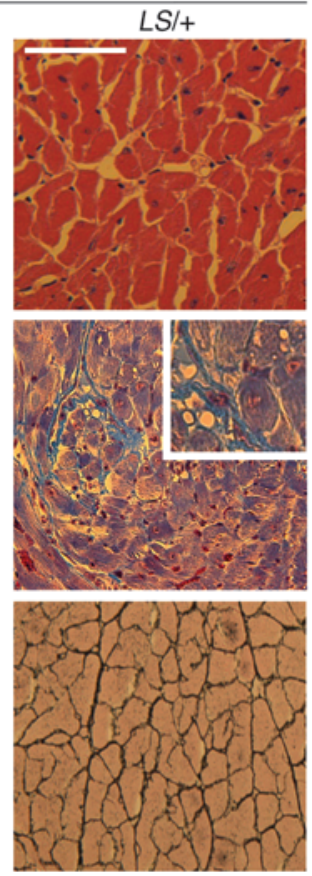

increased diastolic dimensions and thinning of their posterior walls (Figure 5 and Table 2). Left ventricular function in LS/+ mice, as measured by ejection fraction and fractional shortening, also became impaired over time and was statistically different from WT controls at 52 weeks (Table 2).

Cardiac hypertrophy can be secondary to pressure overload caused by stenotic valves. Notably, many NS and some LS patients have valvuloseptal abnormalities $(19,59,60)$. However, $L S /+$ mice had no overt developmental valvular or septal defects, as assessed using histology (data not shown). Cardiac hypertrophy also can occur as a consequence of hypertension; however, arterial blood pressure was

\section{Figure 5}

$L S /+$ mice have HCM that progresses to chamber dilation. Representative echocardiographs of WT and $L S /+$ mice at 12 to 14 weeks of age and 52 weeks of age, respectively. Two-headed arrows indicate left ventricle chamber size. Note that at 12 weeks, $L S /+$ hearts have small chamber dimensions compared with those of WT controls; at 52 weeks, $L S /+$ hearts have increased chamber dimensions and have dilated/decompensated compared with those of WT controls.

\section{Figure 4}

Decreased survival in $L S /+$ mice. (A) Kaplan-Meier curve of WT versus $L S /+$ mice ( $n=5-8$ mice per group at each age point). Note significantly decreased survival in $L S /+$ mice at 12 months. ${ }^{\star} P<0.05$, log-rank test. (B) H\&E-stained sections (original magnification, $\times 400$; scale bar: $100 \mu \mathrm{m}$ ) depicting myofiber disarray and enlarged nuclei in 52-week-old $L S /+$ hearts compared with WT hearts. (C) MassonTrichrome stain of paraffin-embedded longitudinal heart sections from WT and $L S /+$ mice reveals marked fibrosis in $L S /+$ hearts at 52 weeks of age (original magnification, $\times 400$ ). Fibrous tissue is stained in blue and magnified in inset. (D) Reticulin stain of paraffin-embedded heart sections from 52 -week-old WT and $L S /+$ mice (original magnification, $\times 400$ ), showing enlarged $L S /+$ cardiomyocytes.

similar in 8- to 10 -week-old $L S /+$ and WT mice (diastolic blood pressure for $L S /+$ mice, $89.7 \mathrm{mmHg}$, and WT mice, $84.1 \mathrm{mmHg} ; P=0.65$ ). Taken together, our data indicate that the LS-associated Ptpn11 $1279 \mathrm{C}$ mutation causes HCM in mice that over time decompensates to a dilated cardiomyopathy with decreased cardiac function.

LS mutation causes upregulation of cardiac fetal gene expression. Further evidence of the pathological cardiac hypertrophy in $L S /+$ mice was provided by analysis of cardiac fetal gene expression $(3,61)$. In mice, there are 2 differentially expressed cardiac myosin heavy chain (MHC) genes: $\beta-M H C$, which is expressed primarily during fetal development, and $\alpha-M H C$, which is expressed primarily in adult myocardium $(3,61)$. Reexpression of $\beta$-MHC, with a significant transition from $\alpha$-MHC to $\beta$-MHC expression, is a hallmark for cardiac reprogramming and HCM (61). We observed significant expression of $\beta-M H C$ in $L S /+$ hearts compared with that in WT hearts (Figure 6). In addition, there was increased expression of other fetal genes, including atrial natriuretic factor (Anf), brain natriuretic peptide $(B n p)$, and phospholamban $(P l n)$, and decreased expression of the calcium pump, SERCA2A (Figure 6), all of which are consistent with remodeling of the heart and $\operatorname{HCM}(61,62)$.

LS mutants are catalytically impaired and show increased association with Shp2-binding proteins. Previously, we and others reported that LS mutations in SHP2, including Y279C, are catalytically defective and have dominant-negative effects in vitro (39-41). To examine the effect of Shp2-Y279C in vivo, we performed immune complex phosphatase assays using whole heart lysates isolated from WT or $L S /+$ mice that were either treated for 10 minutes with PBS or insulin (10 mU/g body weight). As an additional (positive) control for the sensitivity of our PTP assay, we included lysates from similarly treated D61G/+ mice (43), which express a catalytically activated, NS-associated Ptpn11 allele (Figure 7A). Whereas WT hearts had low basal activity that could be induced by insulin stimulation, $L S /+$ hearts had an approximately $50 \%$ reduction in Shp2 


\section{Table 2}

Anatomic and functional parameters, as assessed by echocardiography, in WT and $L S /+$ mice at the indicated ages

\begin{tabular}{lcccccc} 
& \multicolumn{2}{c}{$\mathbf{4 - 6}$ weeks } & \multicolumn{2}{c}{$\mathbf{1 2 - 1 4}$ weeks } & \multicolumn{2}{c}{$\mathbf{5 2}$ weeks } \\
& WT & LS/+ & WT & LS/+ & WT & LS/+ \\
BW (g) & $19.9 \pm 1.0$ & $18.9 \pm 0.7$ & $24.9 \pm 0.5$ & $22.6 \pm 1.1^{\mathrm{A}}$ & $35.5 \pm 4.5$ & $32.7 \pm 1.8$ \\
HR (bpm) & $658 \pm 21.1$ & $691 \pm 26.9$ & $672 \pm 25.2$ & $648 \pm 25.3$ & $715 \pm 29.1$ & $668 \pm 27.8$ \\
LVPW-th (mm) & $0.93 \pm 1.07$ & $1.15 \pm 0.13$ & $1.04 \pm 0.09$ & $1.58 \pm 0.07^{\mathrm{A}}$ & $0.85 \pm 0.04$ & $0.61 \pm 0.06^{\mathrm{A}}$ \\
LVID-d (mm) & $2.34 \pm 0.16$ & $2.16 \pm 0.10$ & $2.70 \pm 0.23$ & $1.86 \pm 0.24^{\mathrm{A}}$ & $2.83 \pm .086$ & $3.68 \pm 0.15^{\mathrm{A}}$ \\
FS\% & $56.65 \pm 2.29$ & $55.23 \pm 2.28$ & $56.37 \pm 2.33$ & $61.40 \pm 2.35$ & $50.99 \pm 0.23$ & $36.69 \pm 1.94^{\mathrm{A}}$ \\
$n$ & 8 & 8 & 8 & 8 & 5 & 5
\end{tabular}

Note the increased posterior wall thickness, smaller left ventricular chamber dimension, and increased fractional shortening (consistent with enhanced contractility) in 12- to 14-week-old LS/+ hearts compared with that in WT hearts. By 52 weeks, $L S /+$ hearts have thinned and dilated, with functional decompensation, as indicated by decreased fractional shortening. Note that the fractional shortening calculation includes the papillary muscle. No significant differences were seen in any parameters in 4- to 6-week-old $L S /+$ mice compared with WT littermates. Data represent mean \pm SEM; ${ }^{A} P<0.05$, 2-tailed Student's $t$ test (see Supplemental Figure 1 for specific $P$ values). Hr, heart rate; LVID-d, left ventricular chamber dimension in diastole; FS\%, fractional shortening.

phosphatase activity, in accord with the heterozygous expression of the Ptpn11 ${ }^{\mathrm{Y} 279 \mathrm{C}}$ allele. There also was no increase in Shp2 activity after insulin stimulation, consistent with a dominant-negative effect of the LS mutant. By contrast, D61G/+ heart lysates had dramatically enhanced basal and insulin-stimulated Shp2 activity. Similar results were obtained when liver lysates were used as the source for Shp2 (data not shown). Together, these data confirm that Shp2 LS mutations are catalytically defective and have dominant-negative effects in vivo.

Previous in vitro data suggest that LS mutations preferentially adopt the open conformation $(39,41)$. Consistent with this notion, we observed increased basal as well as IGF-1-induced association of Shp2 with IRS-1 (Figure 7B) and Gab1 (data not shown) in LS/+ cardiomyocytes compared with that in WT cells. These data validate our previous results (39) and suggest how LS mutations might function as dominant negatives.

Agonist-evoked Erk activation is impaired in LS/+ mice. Multiple types of receptors contribute to the survival and growth of cardiomyocytes, and SHP2 is required for normal activation of the RAS/MAPK pathway downstream of most, if not all, RTKs, cytokine receptors, GPCRs, and integrins. To assess the functional consequences of LS mutations on Erk activation, we isolated whole hearts from $L S /+$ or WT mice before or 10 minutes after i.p. injection of PBS (vehicle) or insulin. Although we observed no significant difference in basal Erk $1 / 2$ activity between the $L S /+$ and WT hearts, there was significant attenuation in Erk1/2 activation after insulin stimulation in $L S /+$ hearts, compared with WT controls (Figure 8, A and B). Consistent with these data, Erk1/2 activation also was decreased in isolated primary cardiomyocytes from 8-week-old $L S /+$ mice stimulated with RTK (IGF-1, EGF-1), GPCR (angiotensin II), or cytokine (IL-6) agonists (Figure 8C). Together, these data reveal an intrinsic defect in Erk pathway activation in $L S /+$ cardiomyocytes. Moreover, these findings contrast with the effects of an NS-associated, kinase-activating Raf1 mutation in mice, which, as illustrated in the accompanying manuscript (63), develop cardiac hypertrophy due to excessive Ras/Mapk activity (see Discussion).

The Akt/mTor pathway is byperactivated in LS/+ bearts. Shp2 also plays a significant, although complex, role in PI3K/AKT pathway regulation (64-66). Notably, basal as well as insulin-induced Akt ${ }^{473}$ phosphorylation was increased in $L S /+$ hearts (Figure 9, A and B). Basal and agonist-induced $\mathrm{Akt}^{473}$ phosphorylation also was enhanced in IGF-1-stimulated (Figure 9, C and D) or IL-6-stimulated (data not shown) primary cardiomyocytes from $L S /+$ mice, compared with
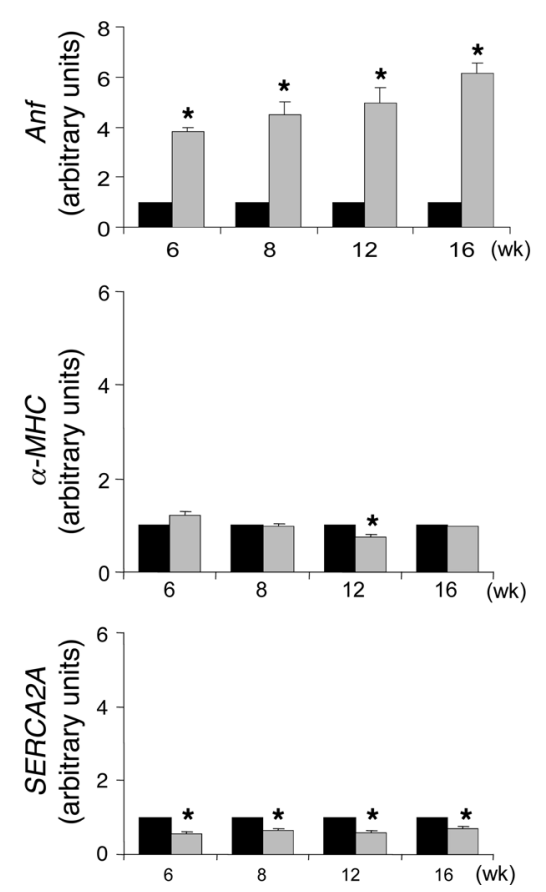

\section{Figure 6}

LS/+ mice upregulate expression of cardiac fetal genes. Total RNA from WT and LS/+ mice ( $n=4-6$ of each genotype) of the indicated ages was used to perform quantitative RT-PCR (each sample in triplicate). The ratio of $\triangle \Delta \mathrm{C}_{\mathrm{T}}$ was analyzed using $G A P D H$ as a control. Data represent mean $\pm \mathrm{SEM}$; ${ }^{*} P<0.01$, 2-tailed Student's $t$ test. Note the significant difference in cardiac fetal gene expression in $L S /+$ and WT hearts of the same age. 
A

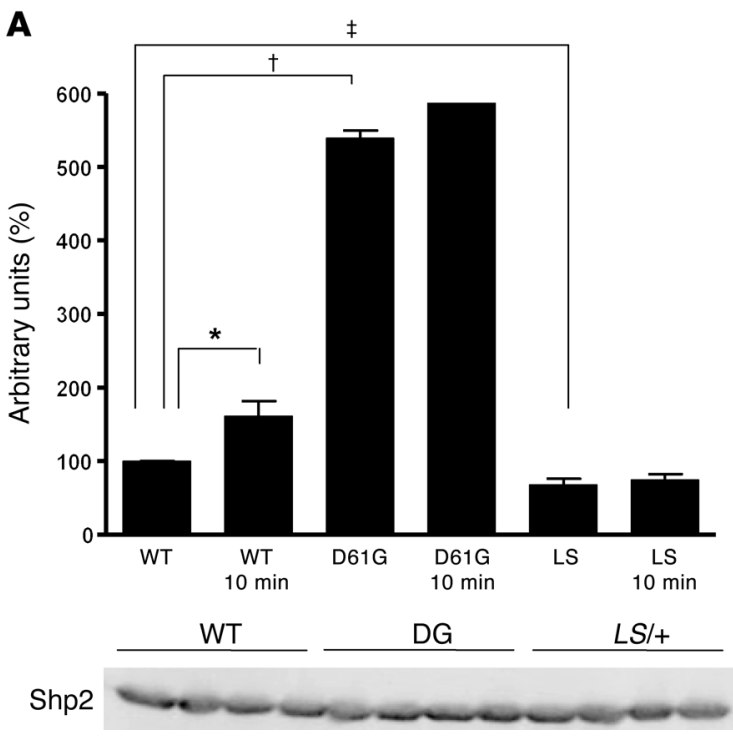

B
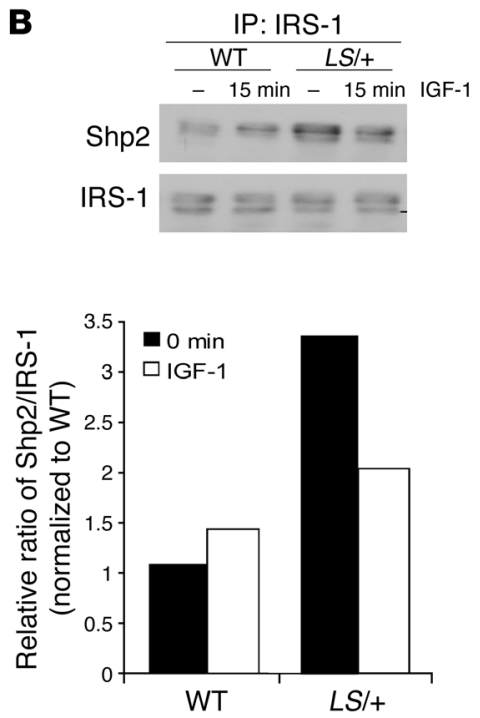

Figure 7

LS mutants are catalytically impaired and show increased binding to IRS-1. (A) WT, DG/+, and LS/+ 8- to 10-week-old mice were treated for 10 minutes with PBS or insulin (10 mU/g body weight). Hearts were isolated and lysed, and Shp2 immune complex PTP assays were conducted using $p$ NPP as a substrate. The bottom panel shows comparable recovery of immunoprecipitated Shp2. Experiments were performed in triplicate ( $n=4-6$ animals per group). Data represent mean $\pm \mathrm{SEM}$; ${ }^{*} P<0.05,{ }^{\dagger} P<0.001$, 邦 $<0.01$. All $P$ values were derived from ANOVA with Bonferroni post-test when ANOVA was significant. (B) Primary cardiomyocytes isolated from 8- to 10-week-old WT or $L S /+$ mice were either left unstimulated or were stimulated for 15 minutes with IGF-1 (10 nM). Lysates from these cells were immunoprecipitated with anti-IRS-1 antibodies and immunoblotted with anti-Shp2 or anti-IRS-1 antibodies, as indicated, to detect complex formation. The bottom panel represents quantification of immunoprecipitated Shp2 normalized to total IRS-1 levels from the above experiment. Note increased IRS-1/Shp2 complex formation in $L S /+$ cardiomyocytes as compared with that in WT cardiomyocytes, even in the absence of IGF-1 stimulation.

that in WT controls. The mTor pathway, a downstream target of Akt, was hyperactivated in $L S /+$ hearts, even under basal conditions, as indicated by elevated levels of Tsc2 phosphorylation at S1462, an Akt phosphorylation site, and enhanced p70S6K phosphorylation (Figure 9C). These data suggested that LS mutants might cause $\mathrm{HCM}$, at least in part, via increased Akt/mTor activation.

The LS/+ mutation impairs multiple downstream signaling pathways involved in HCM. We next analyzed the status of other pathways implicated in cardiac hypertrophy in our $L S /+$ mice. Fak was recently shown to be associated with Shp2 in neonatal rat ventricular myocytes (NRVMs). Depletion of Shp2 by siRNA in these cells increased the phosphorylation of Fak, Akt, Tsc2, and S6 kinase and induced cellular hypertrophy, suggesting that Shp2 can control cardiomyocyte size by downregulation of a pathway that involves Fak and mTor signaling (67). Notably, Fak phosphorylation (at Y397) was significantly elevated in $L S /+$ heart lysates, as compared with that in WT heart lysates (Figure 10, A and B). These data raise the possibility that the elevated levels of Akt phosphorylation in $L S /+$ hearts may be due to increased Fak activity and that Fak might be a substrate for Shp2 in cardiomyocytes (see Discussion).

To assay stress-mediated signaling pathways in $L S /+$ hearts, we measured Jnk1/2 and Stat 3 phosphorylation. Jnk1/2 were constitutively activated in $L S /+$ cardiomyocytes, whereas in WT cardiomyocytes, Jnk1/2 phosphorylation was induced only after IGF-1 (Figure 10C) or IL-6 (data not shown) stimulation. Stat3 phosphorylation also was basally upregulated in $L S /+$ cardiomyocytes, and its phosphorylation increased further after IGF-1 (Figure 10D) or IL-6 (data not shown) stimulation. Together, these data indicate that LS mutants affect several important cellular signaling pathways in the heart. The combined effects of some or all of these signaling abnormalities presumably lead to the cardiac defects associated with LS. $L S /+$ cardiomyocytes respond to rapamycin treatment. Although $L S /+$ mice have alterations in several signaling pathways, the known role of the TOR pathway in the control of cell size $(68,69)$ and the ability of Y279C mutant to enhance Akt/mTor activity, suggested that this pathway might be required for HCM pathogenesis in $L S /+$ mice. To begin to test this possibility, we first asked whether reduction of mTorc 1 activity could ameliorate the cellular abnormalities in $L S /+$ cardiomyocytes. Primary cardiomyocytes from WT or $L S /+$ mice were treated with rapamycin $(0.02 \mu \mathrm{M})$ for 12 hours. After this treatment, the size of the $L S /+$ cardiomyocytes was indeed significantly reduced (Supplemental Figure 2). By contrast, there was no effect of Jnk or Stat inhibitors on overall LS/+ cardiomyocyte cell surface area (Supplemental Figure 2). These data suggested that, while multiple signaling abnormalities might contribute to the cardiac phenotype in $L S /+$ mice, excessive Akt/mTOR signaling is an essential contributor to pathologic cardiac hypertrophy.

We next asked whether we could normalize the cardiomyocyte defects in vivo. We administered daily i.p. injections of either vehicle or rapamycin ( $2 \mathrm{mg} / \mathrm{kg}$ body weight) to 8-week-old WT or $L S /+$ mice for 4 weeks. At the onset of this treatment period, $L S /+$ heart size was not significantly different from that of WT heart size (data not shown, but see Supplemental Figure 1 for reference). When the mice reached 12 weeks of age (a time at which significant hypertrophy is evident in $L S /+$ mice; see Figure 3), we isolated primary cardiomyocytes from vehicle- and rapamycin-treated mice and quantified their area 2 hours after plating. Remarkably, cardiomyocytes 
A

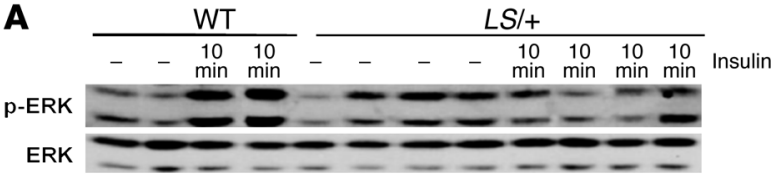

B

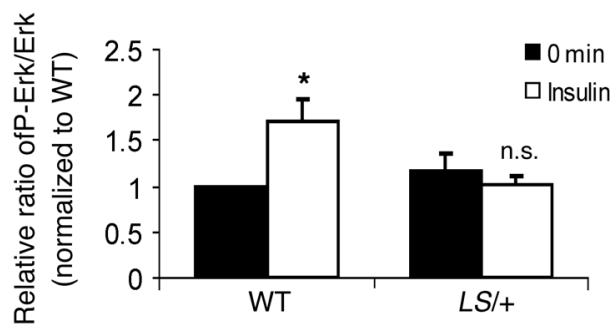

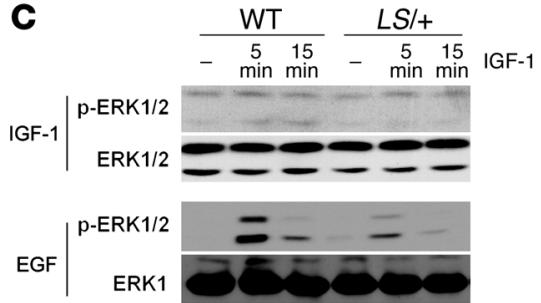

Angll $\mid$\begin{tabular}{r|r}
$\mathrm{p}-\mathrm{ERK} 1 / 2$ & - \\
ERK1 & -
\end{tabular}

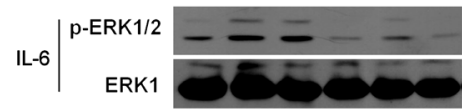

\section{Figure 8}

Agonist-evoked Erk MAPK activation is impaired in $L S /+$ mice. (A) Whole heart lysates from either unstimulated or 10-minute insulin-stimulated (10 mU/g body weight) WT and $L S /+$ mice (8- to 10-week-old) were harvested, lysed, and immunoblotted with anti-phospho-Erk1/2 antibodies. The blot was reprobed with anti-Erk1/2 antibodies as a loading control. Each lane represents an individual animal. (B) Quantification of data collected from 4-6 animals for each group, each derived from 3 different experiments. ${ }^{*} P<0.01$, ANOVA plus Bonferroni post-test when ANOVA was significant. Note the significant difference between basal and insulin-stimulated Erk activity of WT heart lysates, but the lack of significant differences between Erk activity in either basal WT and $L S /+$ hearts or in basal and insulin-stimulated $L S /+$ hearts, respectively. (C) Primary cardiomyocytes isolated from 8-week-old WT and $L S /+$ mice were collected, cultured overnight, and then either left unstimulated or stimulated for the indicated times with IGF-1 (10 nM), EGF $(25 \mathrm{ng} / \mathrm{ml})$, angiotensin II (100 nmol/l), or IL-6 (10 ng/ml). Data are representative of $n=3$ independent experiments. Cell lysates were immunoblotted with anti-phospho-Erk1/2 antibodies, and then membranes were reprobed with anti-Erk1 antibodies to control for loading.

isolated from rapamycin-treated $L S /+$ mice had significantly decreased cell size as compared with that of vehicle-treated $L S /+$ cells (Figure 11A). By contrast, rapamycin (at least at this dose) had little/no effect on the size of WT cardiomyocytes; consequently, the sizes of rapamycin-treated $L S /+$ and WT (untreated or rapamycin-treated) cardiomyocytes were similar (Figure 11B).

As expected, rapamycin treatment reduced the phosphorylation of the downstream mTorc1 target, p70S6 kinase (Figure 11C), but did not affect Erk, JNK, or Stat3 activation (Supplemental Figure 3 ). The phosphorylation of Akt on S473, an mTorc2 phosphorylation site, also was reduced, as was the phosphorylation of GSK-3 $\alpha$ on its Akt site, Ser21. Although rapamycin only inhibits mTorc1 directly, chronic rapamycin administration has been reported to sequester Tor into the (inhibited) mTorc1 complex, resulting in inhibition of both mTor complexes (70). Notably, when we discontinued our daily injections of rapamycin (see below) and administered only a once a week dose of rapamycin, we reversed this effect and normalized Akt activity (data not shown).

Rapamycin treatment normalizes HCM in LS/+ mice. After 4 weeks of treatment, hearts from rapamycin-treated $L S /+$ mice but not vehicle-treated $L S /+$ mice showed a trend toward decreased size, as measured by heart weight to body weight ratios, but this difference did not reach statistical significance (data not shown). Compared with vehicle-treated $L S /+$ mice, rapamycin-treated mice did show a substantial reduction in left ventricular posterior wall thickness (LVPW-th) to values indistinguishable from their WT littermates. However, chamber dimensions remained abnormally small in rapamycin- and vehicle-treated $L S /+$ mice.

These findings indicated that even short-term rapamycin treatment had some therapeutic efficacy for LS-associated HCM and suggested that prolonging the treatment period might be even more beneficial.
Therefore, we treated another cohort of WT and $L S /+$ mice daily with either vehicle or rapamycin ( $2 \mathrm{mg} / \mathrm{kg}$ body weight i.p.) for 4 weeks, followed by an additional 4 weeks of weekly injections of the same dose. A similar protocol has been used to analyze the effects of mTor inhibition in mouse models of polycystic kidney disease (71) and tuberous sclerosis complex disorder (72). Remarkably, after 8 weeks of rapamycin treatment, the gross size of $L S /+$ hearts appeared normal (Figure 12A). LS/+ cardiomyocyte area was reduced, compared with that of vehicle-treated $L S /+$ cells (Figure 12, B and C), to a level comparable to that of WT control cardiomyocytes. Heart weight to body weight ratios of rapamycin-treated $L S /+$ mice decreased significantly, also becoming indistinguishable from control values (Figure 12D). Furthermore, echocardiographic analysis showed normalization of LVPW-th and left ventricular chamber diameter (Figure 12, E and F).

Rapamycin treatment reverses HCM in LS/+ mice. The above experiments indicated that rapamycin treatment could prevent the development of HCM in $L S /+$ mice. To test whether rapamycin also could reverse established hypertrophy, we treated a new cohort of $L S /+$ mice with rapamycin, beginning at 12 weeks of age, when these mice already have apparent HCM (see Figure 3). WT or LS/+ mice were treated daily with vehicle or rapamycin $(2 \mathrm{mg} / \mathrm{kg}$ body weight i.p.) for 4 weeks, followed by an additional 4 weeks of weekly injections of the same dose. Serial echocardiographic analysis at the 4- and 8-week time points (16- and 20-week-old mice, respectively) showed normalization of the LVPW-th and left ventricular chamber diameter (Figure 13, A and B). Furthermore, there was significant reversal of the abnormal fetal gene expression profile of hearts from rapamycin-treated $L S /+$ mice (Figure 14). Together, these data show, that treatment with rapamycin can reverse HCM in $L S /+$ mice and suggest that rapamycin might be an effective, targeted therapy for HCM in human LS patients. 
A
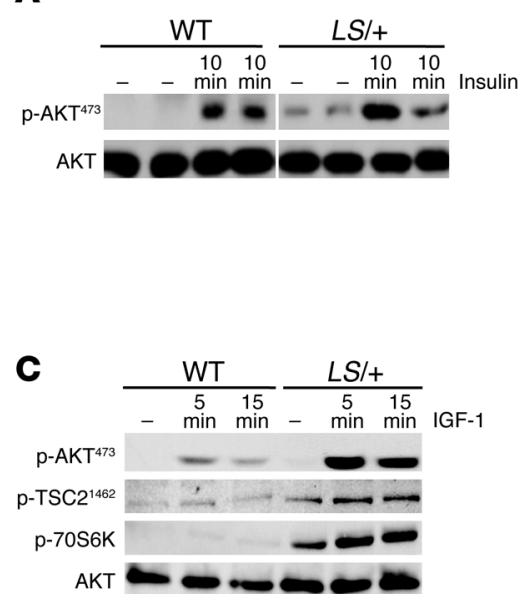

B

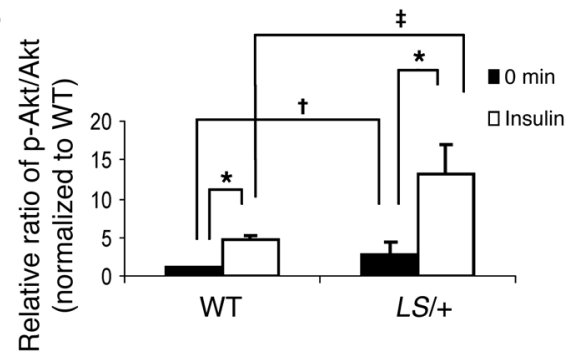

D

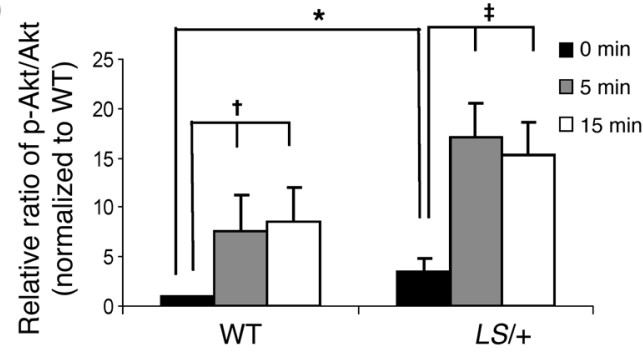

Figure 9

Activation of the Akt/mTOR pathway is enhanced in $L S /+$ hearts and cardiomyocytes. (A) Whole heart lysates from either unstimulated or 10-minute insulin-stimulated (10 mU/g body weight) WT and $L S /+$ mice (8 weeks old) were harvested, lysed, and immunoblotted with antiphospho-Akt ${ }^{473}$ antibodies, followed by anti-Akt antibodies to control for loading. Each lane represents an individual animal. The dividing line represents grouping of images from the same gel. (B) Quantification of data collected from 4-6 animals from each group, derived from 3 inde-

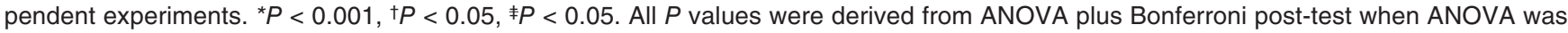
significant. (C) Primary cardiomyocytes from either unstimulated or IGF-1 stimulated (10 nM) WT and $L S /+$ mice (8 to 10 weeks old) were lysed and immunoblotted with anti-phospho-Akt ${ }^{473}, \mathrm{Tsc}^{1462}$, or p70S6K antibodies, as indicated, followed by anti-Akt antibodies to control for loading. (D) Quantification of Akt phosphorylation, derived from 3 independent experiments. ${ }^{\star} P<0.05,{ }^{\dagger} P<0.001$, $\neq P<0.001$. All $P$ values were derived from ANOVA plus Bonferroni post-test when ANOVA was significant.

\section{Discussion}

To study LS pathogenesis, we generated inducible knockin mice harboring the Ptpn11 mutation Y279C. Similar to the human LS phenotype, $L S /+$ mice have short stature, craniofacial dysmorphia, and skeletal abnormalities $(20,31,55)$. In addition, several lines of evidence indicate that $L S /+$ mice have pathological cardiac hypertrophy: (a) their hearts are enlarged, and they show increased heart weight to body weight ratios; (b) $L S /+$ cardiomyocytes show myofiber disarray, inflammatory cells within the myocardial interstitium, and fibrosis; (c) cardiac fetal gene expression is reactivated in $L S /+$ mice, including a shift from $\alpha-M H C$ to $\beta-M H C$ gene expression; (d) $L S /+$ cohorts have an increased death rate compared with that of WT mice; and (e) $L S /+$ mice transition from hypertrophy to dilation by 52 weeks of age, and cardiac function declines concomitantly. Biochemically, $L S /+$ mutant hearts and/or cardiomyocytes show enhanced binding of Shp2 to IRS-1, decreased phosphatase activity, and abrogated agonist-evoked Erk activation, consistent with previous reports that LS mutants have dominant-negative effects in vitro (39-41). By contrast, Akt/mTor signaling is enhanced significantly in $L S /+$ hearts, as well as in cardiomyocytes, consistent with recent findings on fibroblasts isolated from LS patients (73), and there is increased phosphorylation of Stat 3 and Jnk1/2, 2 other signaling molecules implicated in cardiomyopathy $(74,75)$. Although we cannot exclude the possibility that these other signaling pathways contribute to HCM in vivo (i.e., in $L S /+$ mice themselves), inhibiting the mTor pathway by rapamycin treatment completely normalized and reversed the LS cardiac defects, both in vitro and in vivo. Together, these data directly implicate excessive Akt/mTor activity in LS pathogenesis and suggest that therapeutic efficacy of rapamycin should be evaluated in LS patients.
Some features in LS/+ mice are reminiscent of the phenotype of mice expressing NS-associated gain-of-function Ptpn11 mutations $(43,76,77)$ and of the abnormalities seen in NS patients $(78)$. Germline PTPN11 mutations cause NS and LS, and both NS and LS are part of the family of autosomal dominant RASopathies caused by mutations in different components of the RAS/RAF/MEK/ERK MAPK pathway $(29,30)$. Not surprisingly, each of these disorders shares some phenotypes, albeit with varying degrees of penetrance. Yet, each RASopathy also has clinically distinguishable features. Mouse models recapitulated some of these differences. For example, 2 parallel studies on knockin mice expressing the NS-associated Sos1E846K (79) and Raf1-L613V (63) mutations, respectively, show that mutations in different genes along the same pathway yield different phenotypes: Sos1-E846K/+ mice develop HCM with incompletely penetrant aortic stenosis, whereas the Raf1-L613V/+ mice develop HCM with normal valvuloseptal development. Even distinct mutations in the same RASopathy gene can result in distinguishable phenotypes, as shown for Ptpn11 mutations with different degrees of Shp2 activation $(43,76,77)$. Indeed, differential expression of the individual causal mutants, and/or their activity, localization, and feedback regulation in different cell/tissue types, probably accounts for the variable severity and/or phenotype in each RASopathy.

Mutations associated with different RASopathies also have distinct effects in mice. Biochemical, cell biological, and genetic studies indicate that the PTPN11 alleles associated with NS are hypermorphs that enhance Erk pathway activation (44). Given that knockin models of activated Sos1 (79) and Raf1 (63) each show HCM, whereas absence of Ptpn11 in cardiomyocytes causes dilated cardiomyopathy (16), one might expect NS-associated PTPN11 
A

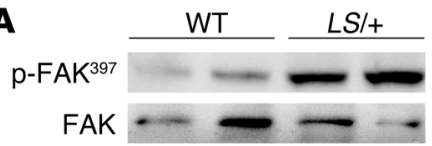

B

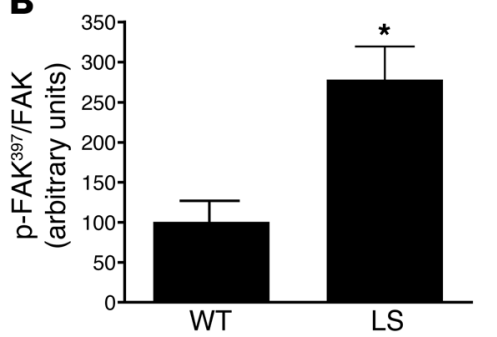

D

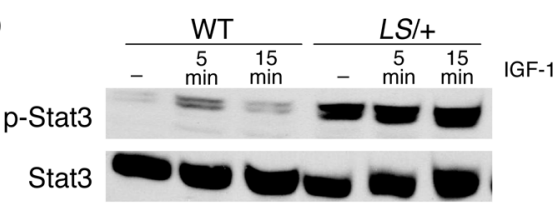

Figure 10

Multiple cardiac signaling pathways are affected in $L S /+$ mice. (A) Whole heart lysates from WT and $L S /+$ 8-week-old mice were harvested, lysed, and immunoblotted with anti-phospho-FAK ${ }^{397}$ antibodies. The blot was reprobed with total anti-Fak antibodies as a loading control. Each lane represents an individual animal. (B) Quantification of data collected from 4-6 animals from each group, derived from 3 independent experiments. ${ }^{*} P<0.05$, 2-tailed Student's $t$ test. (C and D) Primary cardiomyocytes isolated from 8- to 10-week-old WT and $L S /+$ mice were collected, cultured overnight, and then either left unstimulated or stimulated for the indicated times with IGF-1 (10 nM). Cells were lysed and immunoblotted with (C) anti-phospho-JNK1/2 or (D) anti-phospho-Stat3 antibodies. Blots were reprobed with antiJNK or anti-Stat3 antibodies, as indicated, to control for loading. Data are representative of $n=3$ independent experiments.

mutations to cause HCM. Yet, HCM is rare in NS patients with PTPN11 mutations (80-82). Moreover, knockin mice for the NS allele Ptpn11 D61G exhibit valvuloseptal defects similar to those in NS but no evidence of HCM (43). On the other hand, LS is caused almost exclusively caused by catalytically impaired PTPN11 mutations $(39,40)$, and HCM is the most common cardiac abnormality in this syndrome $(34,37,83)$. Our Ptpn $11^{\text {Y279C }}$ knockin mice show that the predominant cardiac manifestation of LS, HCM, is a direct and specific consequence of expression of catalytically impaired mutants of Ptpn11 (rather than, for example, the effects of different modifiers in the human population).

The molecular basis for the events that lead to hypertrophic responses in the heart are confounding and complex. As Shp2 is a critical upstream regulator in multiple RTK, cytokine, GPCR, and integrin-mediated signaling pathways (84), Shp2 mutations might be expected to have several effects in the heart. Indeed, $L S /+$ cardiac lysates show enhanced binding of Shp2 to IRS-1, decreased phosphatase activity, and abrogated agonist-evoked Erk activation, consistent with our previous findings that LS mutants have dominant-negative effects on the Erk/Mapk pathway (39). Notably, 2 previous studies reported that LS mutants evoke increased Erk activation. Expression of the LS mutants Y279C or T468M in Drosophila resulted in ectopic wing veins and a rough eye phenotype, characteristic of increased Erk activity in these tissues, and genetic analysis of enhancers and suppressors also was consistent with hypermorphism of the Erk pathway in these mutant flies (47). Furthermore, in a very recent study, induced pluripotent stem cells (iPSCs) from LS patient-derived fibroblasts showed higher basal p-ERK levels compared with those of control iPSCs (85). By contrast, LS mutants of shp2 were found to have dominant-negative effects when expressed in Zebrafish $(48,49)$, consistent with our findings that basal Erk activation is unchanged and agonist-evoked activation is abrogated in $L S /+$ mice. The reasons 90, 91), supporting our findings that there is a potential regulatory role for mTor in HCM. By contrast, a recent study of transgenic mice with cardiomyocyte-specific expression of constitutively active or kinase-dead mutants of mTor, respectively, showed no overt cardiac phenotype (92). There are several possible explanations for this apparent discrepancy with our findings. First, in the above study, the transgenes were expressed only in cardiomyocytes, whereas in $L S /+$ mice, mutant Ptpn 11 was expressed ubiquitously. Recent data indicate the cardiac fibroblasts can also contribute to HCM (93-96), and it is conceivable that both cardiomyocyte and cardiac fibroblast signaling abnormalities contribute to HCM in our model. Second, transgene expression of the kinase-active and kinase-dead mTor was induced by aMHC-Cre expression, which expresses postnatally in the mouse (97); conceivably, aberrant mTor signaling must occur during development to cause HCM. Third, the extent to which transgenes that drive expression of kinase-dead and kinase-active mTor represent endogenous activation/inactivation of the mTor pathway, as in our $L S /+$ mice, is unclear. For example, kinase-active or kinase-dead mTor would affect mTorc1 and mTorc2. Rapamycin, by contrast, inhibits only mTorc1. Last, as discussed further below, although our experiments using primary cardiomyocytes argue against roles for elevated JNK and/or Stat 3 in cardiac hypertrophy in $L S /+$ mice (Supplemental Figure 3), we cannot (and do not) exclude roles for these other signaling pathways in the whole animal context. Overall, we believe that our system, in which the mTor pathway is activated under endogenous promoter control by the mouse analog of a human disease-associated PTPN11 mutant, is a more physiologically relevant system for analyzing the role of mTor pathway elevation in LS.

Most importantly, our data indicate that the biochemical abnormalities in $L S /+$ hearts have functional consequences, as illustrated by the dramatic effects of rapamycin on the cardiac phenotype of $L S /+$ mice. After 8 weeks of rapamycin treatment, the gross size 
A

A $\quad$ T

WT $\quad$ LS/+ $L S /+$ rapamycin
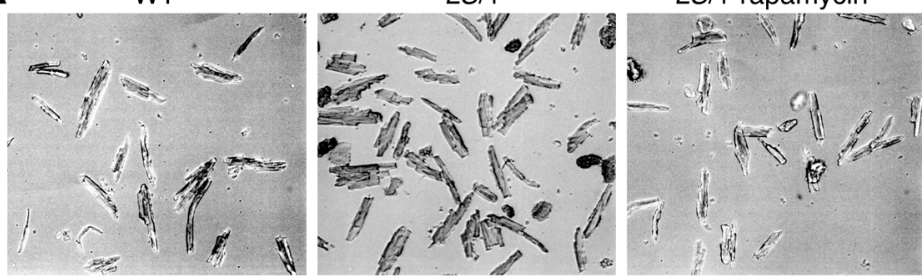

B
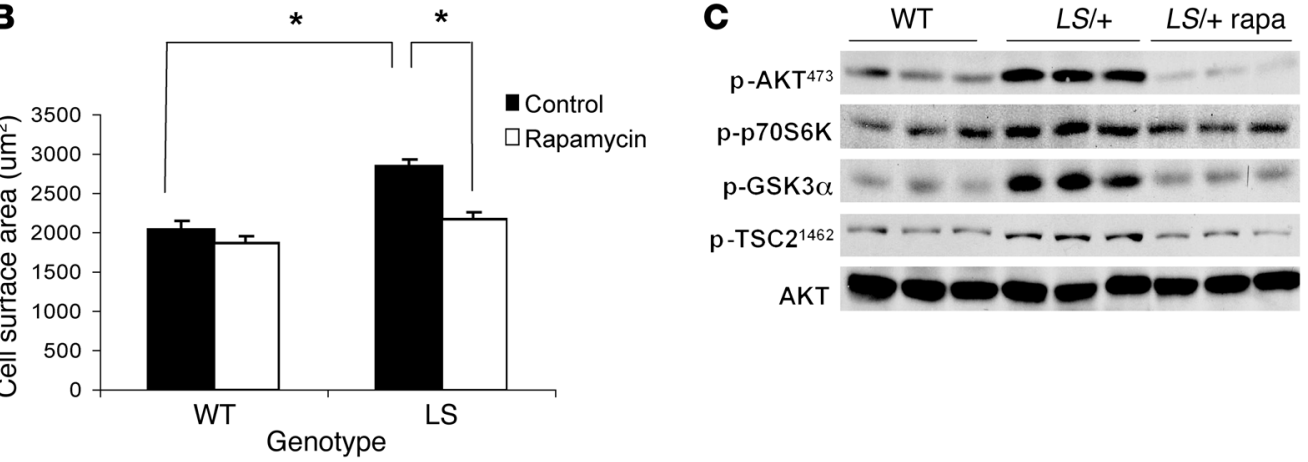

Figure 11

LS/+ cardiomyocytes respond to rapamycin treatment. (A) Photomicrograph of primary cardiomyocytes isolated from vehicle-injected WT, vehicle-injected $L S /+$, or rapamycin-injected $L S /+$ mice after 4 weeks of daily injections. Original magnification, $\times 200$. (B) Quantification of the differences in area between vehicle- or rapamycin-treated WT and $L S /+$ cardiomyocytes (200-500 cells per group). Note the significant difference in total area between WT and $L S /+$ cardiomyocytes. Rapamycin had little/no effect on WT cardiomyocyte cell size. Results are the mean \pm SEM. ${ }^{*} P<0.001$, 2-tailed Student's $t$ test. (C) Inhibiting mTorc1 activity rescues aberrant $L S /+$ signaling. Whole heart lysates from vehicle-treated WT, vehicle-treated $L S /+$, and rapamycin-treated $L S /+$ mice after 4 weeks of daily injections were harvested, lysed, and immunoblotted with antiphospho-Akt ${ }^{473}$, -GSK-3 $\alpha$ (Ser21), -Tsc2 ${ }^{1462}$, and -p70S6K antibodies, followed by anti-Akt antibodies to control for loading, respectively. Note that rapamycin treatment reversed the Akt/mTor pathway signaling defects associated with $L S /+$ mutation. rapa, rapamycin.

of $L S /+$ hearts appeared normal, and cardiomyocyte area, heart weight to body weight ratios, LVPW-th, and left ventricular chamber diameter all were restored to normal levels. At the molecular level, as expected, chronic rapamycin treatment reduced the phosphorylation of Tsc2 and p70S6 kinase. In addition, the phosphorylation of Akt on S473, an mTorc2 phosphorylation site, was reduced, as was the phosphorylation of GSK-3 $\alpha$ on its Akt site, Ser21. Although rapamycin only inhibits mTorc1 directly, chronic rapamycin administration can sequester mTor into the (inhibited) mTorc 1 complex, thereby resulting in inhibition of both mTor complexes (70). Hence, chronic rapamycin treatment might exert its beneficial effects via Akt inhibition as well. However, when we discontinued daily injections of rapamycin and administered it to $L S /+$ mice only once a week, Akt activity was restored to WT levels (data not shown) and their hearts appeared normal (see Figure 12, F and G). Consequently, we suspect that rapamycin's beneficial effects are mediated primarily via mTor inhibition.

Rapamycin is best known as an immunosuppressive drug (98). Nevertheless, it also has been used for other purposes, both in animal models and humans. For example, previous studies have demonstrated its efficacy in the treatment of cardiac hypertrophy in spontaneously hypertensive rats (99) and in reducing pressure overload-induced hypertrophy in mice $(91,100)$. Rapamycin also has proven beneficial for treatment of polycystic kidney disease in mice $(101,102)$, a therapy that has moved to clinical trials $(103,104)$, and a rapamycin analog is now approved for the treatment of renal cancer (105). Taken together, our data argue strongly that clinical trials of rapamycin or a rapamycin analog be considered for the treatment of LS-associated HCM.
Excessive Akt/mTor activity in $\mathrm{LS} /+$ mice is most likely due to increased PI3K activation; indeed, Shp2 can have positive (or negative) effects on the PI3K/Akt pathway, depending on the specific upstream pathway and/or the cellular context (66). There are at least 2 potential ways in which LS mutants might cause increased PI3K activation. First, previous work has suggested that Shp 2 can dephosphorylate the binding sites for PI3K on Gab1 $(66,106)$. Recent studies of the effects of Ptpn11 deletion in the liver suggest that Shp2 also dephosphorylates the PI3K binding sites on IRS-1/2 (107). Alternatively, or in addition, excessive Fak activity may also play a role in increased PI3K activation in $L S /+$ hearts. A recent study showed that Fak is associated with Shp2 in NRVMs. Moreover, depletion of Shp2 by siRNA increased phosphorylation of Fak, Akt, Tsc2, and S6 kinase and induced hypertrophy of NRVMs (67). Indeed, heart lysates from our $L S /+$ mice had elevated Fak phosphorylation as well. Further studies will be required to determine whether, in fact, $L S /+$ hearts/ cardiomyocytes have increased PI3K activity and, if so, whether either (or both) of the above mechanisms is responsible for this increase.

While it is true that the PI3K/Akt pathway plays an important role in regulating cardiac homeostasis/adaptive hypertrophy (108), the mechanisms are controversial. Mice expressing a cardiac-specific constitutively active mutant of the PI3K $110 \alpha$ subunit developed substantial physiological hypertrophy but did not transition into pathological hypertrophy (109). Conversely, cardiac expression of a dominant-negative PI3K p $110 \alpha$ catalytic subunit impaired physiological hypertrophy $(110,111)$, whereas the PI3K p110 $\gamma$ subunit was shown to be required for maladaptive hypertrophy $(111,112)$. On the other hand, short-term activation of Akt 1 promotes cardiomyocyte viability and reverses hypertrophy, while 

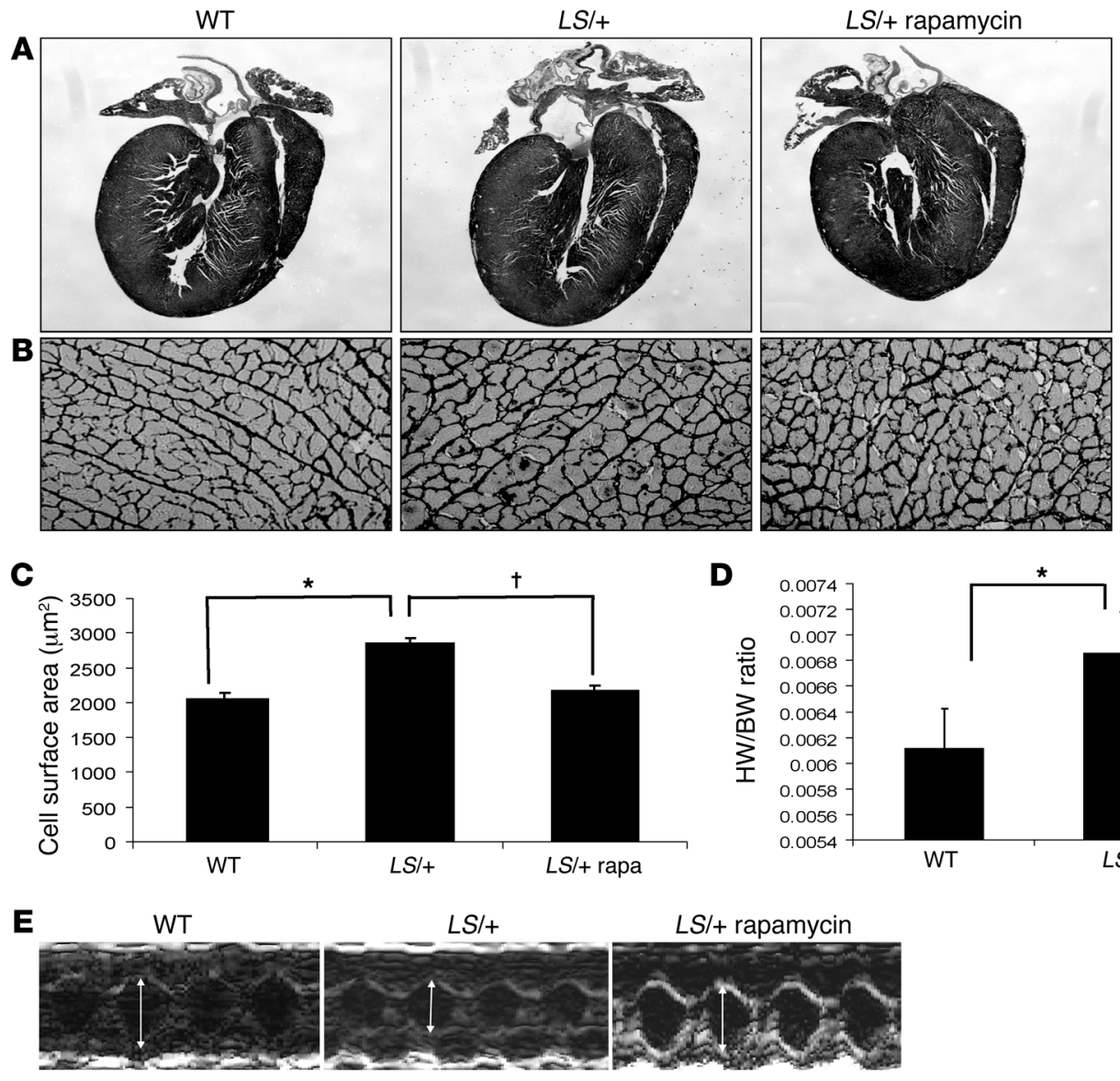

$\mathbf{F}$
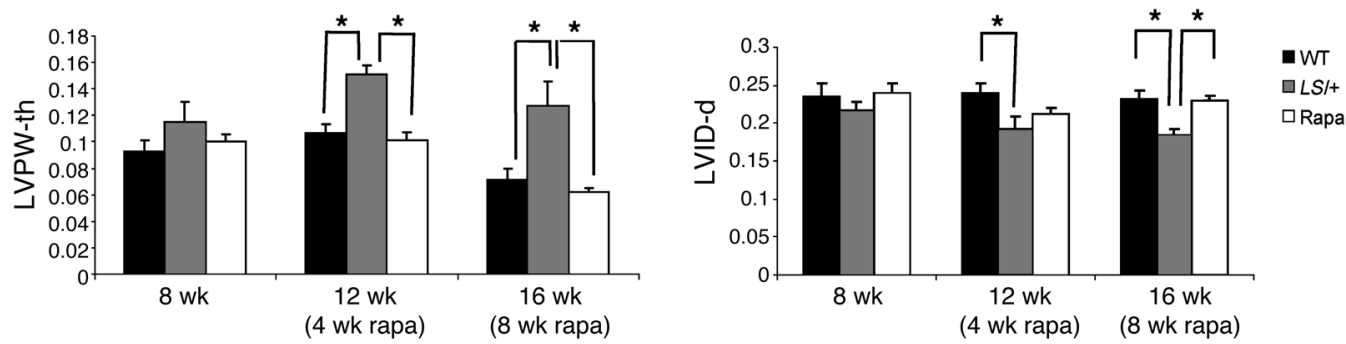

Figure 12

Rapamycin normalizes HCM in LS/+ mice. (A) H\&E-stained longitudinal sections of hearts from WT and LS/+ mice. Note normalization of hypertrophy in $L S /+$ hearts after rapamycin treatment (original magnification, $\times 100$ ). (B) Reticulin stain of paraffin-embedded heart sections from 16-week-old WT and $L S /+$ mice (original magnification, $\times 400$ ). (C) Quantification of average area (in $\mu^{2}{ }^{2}$ ) of cardiomyocytes (200-500 cells counted/group) from WT or $L S /+$ cardiomyocytes isolated from mice that were either vehicle- or rapamycin-treated (2 mg/kg body weight) daily by i.p. injection for 4 weeks, then weekly for 4 weeks; see Results and Methods for details. Results are shown as the mean \pm SEM. ${ }^{*} P<0.05$, ${ }^{\dagger} P<0.05$. (D) Heart weight to body weight ratios of WT and $L S /+$ mice with vehicle- or rapamycin-treatment, as indicated. ${ }^{*} P<0.05,{ }^{\dagger} P<0.001$. (E) Representative echocardiographs from 16-week-old vehicle-treated WT, vehicle-treated $L S /+$, or rapamycin-treated $L S /+$ mice. Two-headed arrows indicate left ventricle chamber size. (F) Anatomic and functional parameters of 12- and 16-week-old WT, $L S /+$, and $L S /+$ rapamycintreated mice, as assessed by echocardiography, either after 4 weeks (measured at 12 weeks of age) or after 4 weeks of daily i.p. injections, followed by 4 weeks of weekly (measured at 16 weeks of age) i.p. injections. LVID-d, left ventricular chamber dimension in diastole. ${ }^{*} P<0.001$ denotes significance between the vehicle-treated 16 -week-old $L S /+$ mice and the $L S /+$ rapamycin-treated mice. All $P$ values in $\mathbf{C}, \mathbf{D}$, and $\mathbf{F}$ were derived from ANOVA and Bonferroni post-test when ANOVA was significant.

chronic Akt1 activation induces extensive cardiac hypertrophy, with severe contractile dysfunction and massive interstitial fibrosis $(113,114)$. These findings indicate that the PI3K/Akt pathway is highly complex, with differential roles in regulating cardiac hypertrophy. Clearly, further studies are needed to better elucidate the mechanism(s) for these signaling-specific differences.
Indeed, although our data clearly implicate excessive Akt/ mTor activity in LS cardiac pathogenesis, we cannot and do not exclude the possibility that other signaling abnormalities contribute either to the initial hypertrophic phase or to the ultimate dilation of LS hearts. For example, the inability of LS mutants to activate Erk in response to agonist stimula- 
A

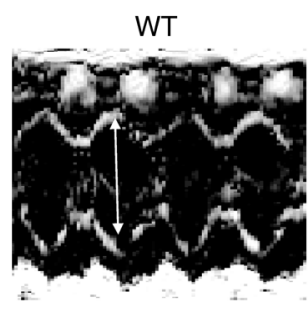

WT rapamycin

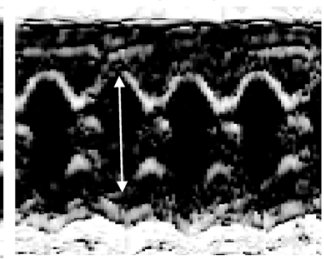

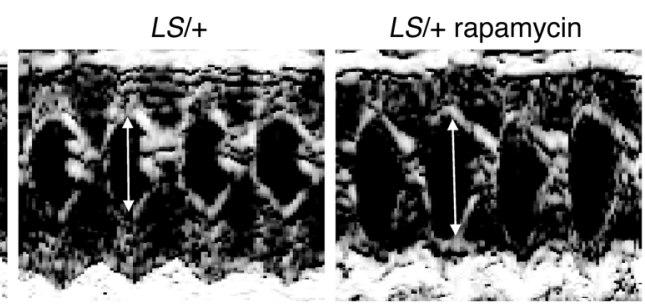

B
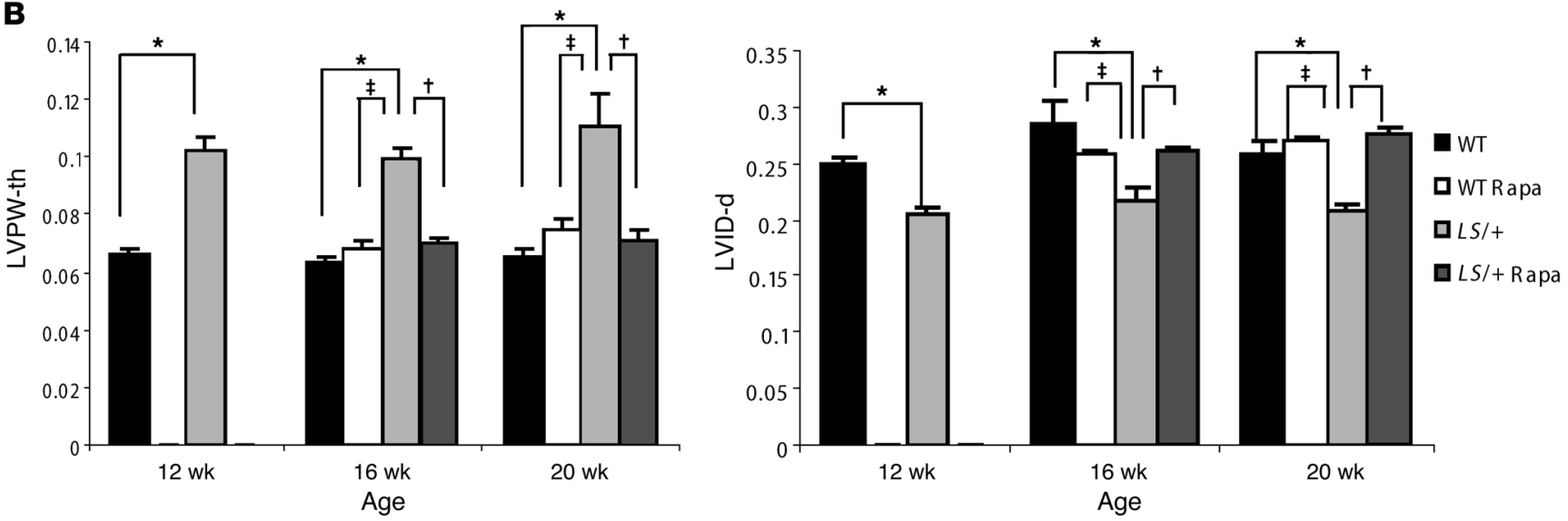

Figure 13

Rapamycin reverses HCM in LS/+ mice. (A) Representative echocardiographs from 16-week-old vehicle-treated WT, rapamycin-treated WT, vehicle-treated $L S /+$, or rapamycin-treated $L S /+$ mice. Two-headed arrows indicate left ventricle chamber size. (B) Anatomic and functional parameters of 12-, 16-, and 20-week-old WT, rapamycin-treated WT, LS/+, and rapamycin-treated LS/+ mice, as assessed by echocardiography, either after 4 weeks (measured at 16 weeks of age) or after 4 weeks of daily i.p. injections, followed by 4 weeks of weekly (measured at 20 weeks of age) i.p. injections. ${ }^{*} P<0.001,{ }^{\dagger} P<0.001$, ${ }^{\ddagger} P<0.001$. All $P$ values were derived from ANOVA and Bonferroni post-test when ANOVA was significant.

tion might contribute to the eventual transition to dilation in the $L S /+$ mice, perhaps in tandem or in parallel with increased Jnk and/or Stat3 activities. Jnk and/or Stat3 themselves might be important contributors to hypertrophy in LS; increased Jnk activity is associated with development of cardiac hypertrophy (74), and Stat3 phosphorylation is significantly upregulated in the failing heart with dilated cardiomyopathy (75). Interestingly, however, inhibition of Jnk and Stat3 had little effect on overall cardiomyocyte cell surface area in vitro (Supplemental Figure 2). Given that these inhibitors lack specificity and have poor bioavailability, evaluation of the overall contribution of aberrant Jnk and Stat activity to HCM in LS/+ mice (in vivo) will require genetic approaches. However, it will be important to clarify the role of these other signaling abnormalities in LS pathogenesis, as they may provide additional targets for therapeutic intervention.

In summary, our data support the conclusion that excessive Akt/mTOR activity is essential for the development and maintenance of HCM in LS, and we identify a potential effective treatment for the reversal of HCM in LS patients. Attention has naturally focused on the ERK/MAPK pathway in explaining the pathogenesis of RASopathies. However, our data argue that different RASopathies can have complex and distinct effects on cellular signaling pathways. Knowledge of the detailed effects of these mutations on molecular and cellular signaling is essential for a more rational and personalized approach to therapy for these disorders.

\section{Methods}

\section{Generation of LS/+ mice}

The targeting construct was generated using recombineering methodology as developed by Liu et al. (51). Our strategy used unidirectional Cre-mediated inversion of a duplicated mutant (Y279C) exon, permitting expression of the WT protein in the absence of Cre and the mutant product after Cre exposure. Briefly, a 9.3-kb fragment (based on the C57BL/6 genome, build NCBIM37) of the mouse Ptpn11 gene containing exons 7-9 (extending 4,871 bp upstream of exon 7 and 2,148 bp downstream of exon 9) was subcloned by recombineering from the bacterial artificial chromosome, bMQ-311k06 (Geneservice), into a plasmid containing a TK selection cassette. A loxP site was inserted by recombination $371 \mathrm{bp}$ upstream of exon 7. Exon 7 was amplified by PCR (761-bp fragment extending 371 bp upstream and 294 bp downstream), and a single base change $(A>C)$ was introduced into the codon for amino acid 279 (Y279C) by site-directed mutagenesis. The point mutation and surrounding region were sequenced to verify the mutation and the fidelity of the amplification and then were subcloned into a modified neo cassette, consisting of a loxP511 site, the inverted mutant exon fragment, an inverted loxP site, an inverted loxP511 site, and a neo selection cassette flanked by FRT sites. This cassette was inserted by recombineering $294 \mathrm{bp}$ downstream of exon 7 to generate final targeting construct.

This construct was linearized with NotI, electroporated into TC1 (129S6/SvEv) ES cells, and selected with G418 and FIAU. Resistant colonies were expanded and screened by long-range PCR, using primers outside and inside the targeting vector to identify homologous recombinants (see Supplemental Table 1). Recombinant clone 1F10 was used 

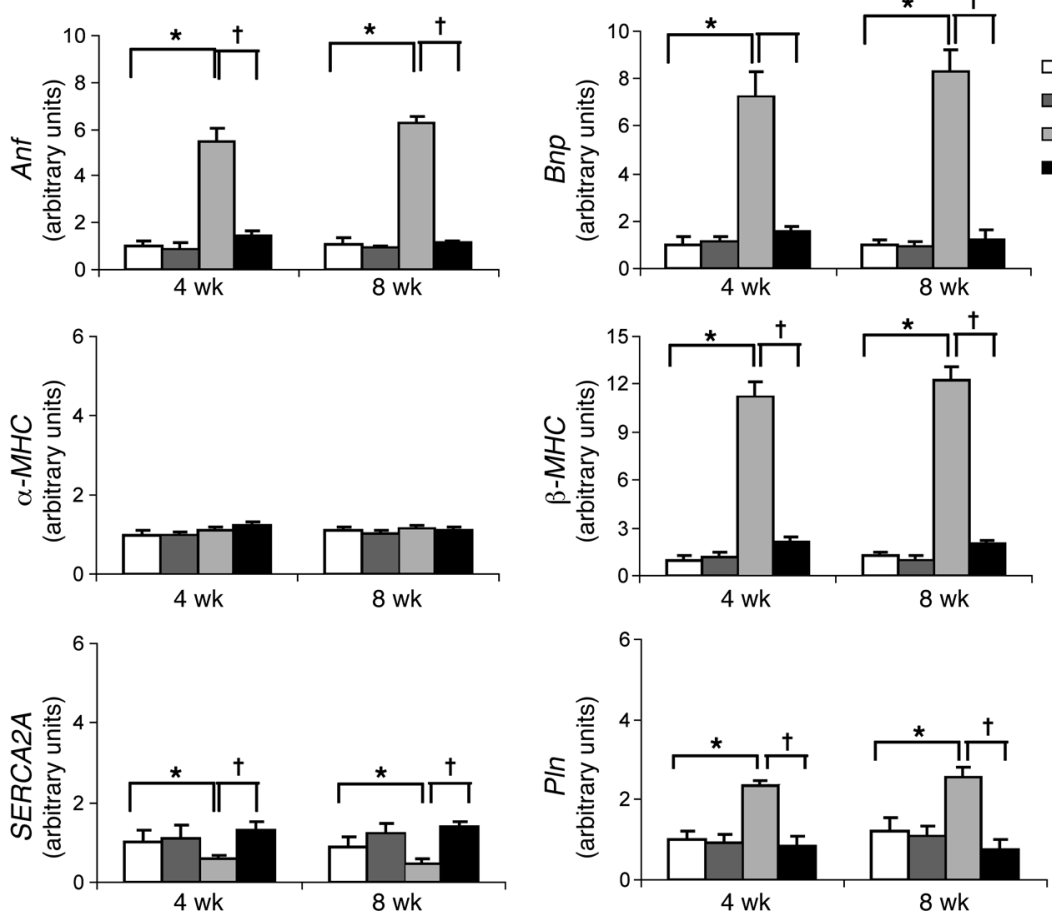

\section{Figure 14}

Rapamycin treatment normalizes cardiac fetal gene expression in $L S /+$ mice. Total RNA from vehicle- or rapamycin-treated WT and $L S /+$ mice ( $n=4-6$ of each genotype) of the indicated ages was used to perform quantitative RT-PCR (each sample in triplicate). The ratio of $\Delta \delta \mathrm{C}_{\mathrm{T}}$ was analyzed using Gapdh as a control. Note that rapamycintreated $L S /+$ heart lysates have normalized fetal gene expression profiles as compared with those of WT heart lysates. Data represent mean \pm SEM. ${ }^{*} P<0.01,{ }^{\dagger} P<0.001$, 2-tailed Student's $t$ test. for blastocyst injection. Chimeras were generated (Brigham and Women's Transgenic Core Facility), and germline transmission of $i L S /+$ mice was obtained. iLS/+ (129S6/SvEv $\times$ C57BL6/J) mice were crossed to Frt-Flp (C57BL6/J) mice (The Jackson Laboratory) to excise the neo cassette. iLS/+ mice with excised neo cassette were then crossed to EIIA-Cre (C57BL6/J) mice (The Jackson Laboratory), which express Cre ubiquitously, and then to WT (C57BL6/J) mice to generate mice with global LS Y279C expression ( $L S /+$ mice). Subsequent generations of $L S /+$ mice were crossed to WT $\mathrm{C} 57 \mathrm{BL} / \mathrm{J}$ mice, thereby maintaining the line on a C57BL6/J background; these mice were used for all experiments. Genotyping was carried out by PCR using tail DNA (see Supplemental Table 1). Unless otherwise indicated, WT C57BL6/J littermates were used as controls.

All animal studies were approved by the Beth Israel Deaconess Medical Center IACUC Committee and performed in accordance with IACUC standards. $\mu \mathrm{CT}$ scans (conducted at the Ontario Cancer Institute) were approved by the University Health Network Animal Care Committee and performed in accordance with the standards of the Canadian Council on Animal Care.

\section{Body size analysis and morphometry}

For growth curves, body length (anal-nasal length) and weight were measured biweekly. For skeletal morphometry using $\mu \mathrm{CT}$, mice were anesthetized with $2 \%$ isoflurane and scanned using a Locus Ultra MicroCT Scanner (GE Healthcare). Three-dimensional images of the skeleton were generated and analyzed with GEHC MicroView Software (GE Healthcare). Skull measurements were made according to the Standard Protocol and Procedures from The Jackson Laboratory (http://craniofacial.jax.org/standard_protocols.html).

\section{Histology}

Hearts for morphometry and histochemistry were flushed with PBS, perfusion fixed in formalin or Bouin's reagent, and paraffin embedded. Sections $(5 \mu \mathrm{m})$ were stained with H\&E, Masson-Trichrome, or reticulin at the Rodent Histopathology Core at Harvard Medical School. Crosssectional length, width, and area of cardiomyocytes with centrally located nuclei (to ensure the same plane of sectioning) were measured using ImageJ 1.41 software (developed by Wayne Rasband; http://rsbweb.nih.gov/ij/). Five individual samples were analyzed for each genotype, with 200-500 cells measured in each.

\section{Electron microscopy}

Electron microscopy was performed at the Harvard Medical School Facility. Briefly, mouse hearts were perfused in PBS and fixed with a mixture of paraformaldehyde $(2 \%)$ and glutaraldehyde $(1.5 \%)$ in cacodylate buffer $(0.1 \mathrm{M}$ $\mathrm{Na}$ cacodylate, $\mathrm{pH}$ 7.4). Pieces $\left(1 \mathrm{~mm}^{3}\right)$ were dissected from the left ventricle, postfixed in $1 \% \mathrm{OsO}_{4}$, dehydrated in a series of alcohols, and embedded in Epon Araldite (SPI Supplies). Ultrathin sections (silver to gold) were obtained with a Reichert Ultracut E microtome, stained with uranyl acetate and lead citrate, and observed in a Tecnai $\mathrm{G}^{2}$ Spirit BioTWIN (Tecnai), with an AMT 2k charge-coupled device camera.

\section{Quantitative real-time PCR}

RNA was isolated from whole hearts with TRIzol (Invitrogen) or using the RNeasy Mini Kit (QIAGEN). Quantitative real-time PCR was performed with SYBR Green (Applied Biosystems), using an Applied Biosystems 7900. Gene expression analyses for $\alpha-M H C, \beta-M H C, A n f, B n p, S E R C A 2 A$, and $P l n$ were carried out according to the manufacturer's instructions. Primer sequences and conditions are listed (Supplemental Table 2). Each measurement was obtained using at least 3 mice per group, and each sample was measured in triplicate. Data were quantified by using the comparative $\mathrm{C}_{T}$ method, with Gapdh expression as a control.

\section{Echocardiography}

Transthoracic echocardiography was conducted on nonanesthetized animals as described previously (115), with a 13-MHz probe (Vivid 7, GE Medical Systems) or VisualSonics Vevo 770 high-frequency ultrasound rodent imaging system. GE Medical Systems or VisualSonics Vevo 770 software was used for data acquisition and subsequent analysis. Hearts were imaged in the 2-dimensional parasternal short-axis view, and an 
M-mode echocardiogram of the midventricular region was recorded at the level of the papillary muscles. Calculations of cardiac anatomic and functional parameters were carried out as described previously (115).

\section{Hemodynamic studies}

Cardiac function was evaluated by measuring the maximum rate of increase $(d P / d t \max )$ and decline $(d P / d t$ min) in left ventricular pressure with a micromanometer catheter (Millar 1.4F, SPR 671, Millar Instruments) positioned in the left ventricle via a right common carotid artery cannulation.

\section{Biochemical analyses}

Whole hearts from WT or $L S /+$ mice were dissected, perfused in PBS, and immediately frozen in liquid $\mathrm{N}_{2}$. Whole-cell lysates were prepared by homogenizing the tissue in radioimmunoprecipitation (RIPA) buffer $(25 \mathrm{mmol} / \mathrm{l}$ Tris-HCl [pH 7.4], 150 mmol/1 NaCl, $0.1 \%$ SDS, $1 \% \mathrm{NP}-40,0.5 \%$ sodium deoxycholate, $5 \mathrm{mmol} / 1 \mathrm{EDTA}, 1 \mathrm{mmol} / 1 \mathrm{NaF}, 1 \mathrm{mmol} / 1$ sodium orthovanadate, and a protease cocktail) at $4^{\circ} \mathrm{C}$, followed by clarification at $14,000 \mathrm{~g}$.

Primary cardiomyocytes were isolated and cultured as previously described (116). Briefly, hearts were placed in a Langendorff apparatus, and adult cardiomyocytes were obtained by perfusion with $\mathrm{Ca}^{2+}$-free Tyrode buffer (135 mmol/1 NaCl, $4 \mathrm{mmol} / \mathrm{l} \mathrm{KCl,} 1 \mathrm{mmol} / \mathrm{l} \mathrm{MgCl}, 0.33 \mathrm{mmol} / 1 \mathrm{NaH}_{2} \mathrm{PO}_{4}$, $10 \mathrm{mmol} / \mathrm{l} \mathrm{HEPES}$ ) (pH 7.4) and $10 \mathrm{mmol} / \mathrm{l}$ glucose, $10 \mathrm{mmol} / 1$ 2,3-butanedione monoxime (Sigma-Aldrich), and $5 \mathrm{mmol} / 1$ Taurine (Sigma-Aldrich) for 3 to 5 minutes. Perfusion was continued for an additional 7 to $10 \mathrm{~min}$ utes with recirculating Tyrode buffer, containing a mixture of collagenase $\mathrm{D}$ ( $0.3 \mathrm{mg} / \mathrm{g}$ body weight; Roche), collagenase B $(0.4 \mathrm{mg} / \mathrm{g}$ body weight; Roche), and proteinase XIV $(0.05 \mathrm{mg} / \mathrm{g}$ body weight; Sigma-Aldrich). Ventricular tissue was then minced in Tyrode solution containing $2 \%$ bovine serum albumin (Sigma-Aldrich), incubated for 15 minutes at $37^{\circ} \mathrm{C}$, and filtered through a $250-\mu \mathrm{m}$ nylon mesh. The cell suspension was centrifuged at $420 \mathrm{~g}$ for 2 minutes and then gradually subjected to Tyrode buffer containing increasing concentrations of calcium and decreasing concentrations of 2,3-butanedione monoxime (i.e., the final concentration was $1.2 \mathrm{mmol} / \mathrm{l} \mathrm{CaCl}_{2}$ with no 2,3-butanedione monoxime). Typical yields were $1.5 \times 10^{6}$ to $2.5 \times 10^{6}$ cells per heart, with $70 \%$ to $80 \%$ of the cells retaining their rod-shaped morphology. After 12 hours, cardiomyocytes were stimulated for the indicated times with the appropriate agonist ( $10 \mathrm{nmol} / \mathrm{l}$ IGF-1, $25 \mathrm{ng} / \mathrm{ml} \mathrm{EGF,} 100 \mathrm{nmol} / \mathrm{l}$

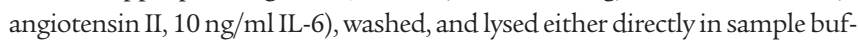
fer containing SDS or in RIPA at $4^{\circ} \mathrm{C}$, followed by clarification at $14,000 \mathrm{~g}$.

After 12 hours, cardiomyocytes were stimulated for the indicated times with

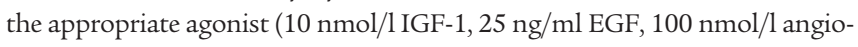
tensin II, or $10 \mathrm{ng} / \mathrm{ml} \mathrm{IL6),} \mathrm{washed,} \mathrm{and} \mathrm{either} \mathrm{lysed} \mathrm{in} \mathrm{RIPA} \mathrm{buffer} \mathrm{at} 4^{\circ} \mathrm{C}$, followed by clarification at $14,000 \mathrm{~g}$, or directly in SDS-PAGE sample buffer. Proteins were resolved by SDS-PAGE and transferred to PVDF membranes. Immunoprecipitations were performed with either anti-Gab1 or anti-IRS-1 antibodies (Cell Signaling Technology). Immunoblots were performed, following the manufacturer's directions, with anti-Akt, anti-JNK, anti-GSK-3 $\alpha$, anti-phospho-FAK-297, or anti-Shp2 antibodies (all from Santa Cruz Biotechnology Inc.); anti-Stat3, anti-Erk1/2, anti-Gab1, anti-IRS-1, anti-TSC2 ${ }^{1462}$, or anti-p70S6 kinase antibodies (all from Cell Signaling Technology); or antiphospho-Akt ${ }^{473}$, anti-phospho-Erk1/2, anti-phospho-Stat 3, anti-phosphoJNK, anti-phospho-TSC2 ${ }^{1462}$, anti-phospho-p70S6 kinase, or anti-phosphoGSK-3 $\alpha$ antibodies (all from Cell Signaling Technology). Bands were visualized with enhanced chemiluminescence and quantified by densitometry (developed by Wayne Rasband; ImageJ 1.41 software, http://rsbweb.nih.gov/ij/).

\section{Immune complex PTP assays}

PTP assays were conducted as previously described (39) using paranitrophenyl phosphate ( $p$ NPP, obtained from Sigma-Aldrich) as substrate. WT or $L S /+$ whole heart lysates were homogenized and lysed in RIPA buffer (but without sodium orthovanadate), and Shp2 was immunoprecipitated by using anti-Shp2 polyclonal antibodies (Santa Cruz Biotechnology Inc.) coupled to protein A-Sepharose. Immune complexes were washed 3 times in RIPA buffer without sodium orthovanadate and once in wash buffer (30 mm HEPES [pH 7.4], 120 $\mathrm{mm} \mathrm{NaCl}$ without $p \mathrm{NPP}$ ). For each sample, PTP assays were performed in triplicate at $37^{\circ} \mathrm{C}$ in $50 \mu \mathrm{l}$ assay buffer (30 mm HEPES [pH 7.4], $120 \mathrm{~mm} \mathrm{NaCl}, 5 \mathrm{~mm}$ dithiothreitol, $10 \mathrm{~mm}$ pNPP) containing $50 \mu \mathrm{l}$ of the Shp2 beads. Reactions were terminated with $0.2 \mathrm{~N} \mathrm{NaOH}$, and phosphate release was determined by measuring $A_{410}$. After the assays, immune complexes were recovered by centrifugation, boiled in $2 \times$ SDSPAGE sample buffer, resolved by SDS-PAGE, and immunoblotted with polyclonal Shp2 antibodies (Santa Cruz Biotechnology Inc.) to ensure that equal amounts of Shp2 had been tested for phosphatase activity.

\section{Rapamycin studies}

In vitro. When indicated, primary cardiomyocytes were treated in vitro with rapamycin (Sigma-Aldrich) dissolved in DMSO at $0.02 \mu \mathrm{M}$ or DMSO alone for 12 hours.

In vivo. Rapamycin (LC Laboratories) was dissolved in ethanol at a concentration of $20 \mathrm{mg} / \mathrm{ml}$, filter sterilized, resuspended in vehicle $(0.25 \% \mathrm{PEG}$, $0.25 \%$ Tween-80) at a concentration of $1 \mathrm{mg} / \mathrm{ml}$, and injected i.p. $(2 \mathrm{mg} / \mathrm{kg}$ body weight), either daily for 4 weeks or daily for 4 weeks followed by weekly injections for an additional 4 weeks. Injections began at either 8 weeks (prior to onset of hypertrophy) or 12 weeks (after established hypertrophy was indicated) of age, and mice were assessed after 4 weeks of treatment or after 8 weeks of treatment, as detailed in the Results and figure legends. As controls, WT and $L S /+$ mice were treated with vehicle alone.

In vitro Jnk and Stat 3 inhibition experiments. When indicated, similarly to rapamycin treatment, primary cardiomyocytes were treated in vitro with either Jnk Inhibitor II (Calbiochem) or Stat Inhibitor V (Santa Cruz) dissolved in DMSO at $10 \mu \mathrm{M}$ and $1 \mu \mathrm{M}$, respectively.

\section{Statistics}

All data are expressed as mean \pm SEM. Statistical significance was determined using 2-tailed Student's $t$ test and 1-way ANOVA or 2-way repeated measure ANOVA, as appropriate. If ANOVA was significant, individual differences were evaluated using the Bonferroni post-test. Kaplan-Meier survival curves were analyzed using the log-rank test. For all studies, values of $P<0.05$ were considered statistically significant.

\section{Acknowledgments}

We thank Clare Malone and Sotirios Banakos from the Kontaridis laboratory for their technical assistance related to the work in this manuscript. We would also like to thank Glenn Rowe and Matthew Coggins for their assistance with RT-PCR and echocardiography, respectively. This work was supported by NIH grants HL088514 (to M.I. Kontaridis) and HL083273 (to B.G. Neel), the São Paulo Research Foundation (FAPESP 2006/55920 to T.M. Marin), and the Frederick Banting and Charles Best Canada Graduate Scholarship (to X. Wu). This work was also supported in part by the Milton Fund and the Beth Israel Deaconess Medical Center Division of Cardiology (to M.I. Kontaridis) and in part by the Ontario Ministry of Health and Long-Term Care (to B.G. Neel and X. Wu). The views expressed do not necessarily reflect those of the Ontario Ministry of Health and Long-Term Care or Beth Israel Deaconess Medical Center.

Received for publication November 28, 2009, and accepted in revised form August 31, 2010. 
Address correspondence to: Maria I. Kontaridis, Department of Medicine, Division of Cardiology, Beth Israel Deaconess Medical Center, Center for Life Sciences, Room 908, 3 Blackfan Circle, Boston, Massachusetts 02115, USA. Phone: 617.735.4248; Fax: 617.735.4255; E-mail: mkontari@bidmc.harvard.edu.
Or to: Benjamin G. Neel, Ontario Cancer Institute and Princess Margaret Hospital, University Health Network, MaRS Centre, Toronto Medical Discovery Tower, 8th Floor, Rm 8-601, 101 College Street, Toronto, Ontario, Canada M5G 1L7. Phone: 416.581.7710; Fax: 416.581.7698; E-mail: bneel@uhnresearch.ca.
1. Weismann CG, Gelb BD. The genetics of congenital heart disease: a review of recent developments. Curr Opin Cardiol. 2007;22(3):200-206.

2. Eisenberg LM, Markwald RR. Molecular regulation of atrioventricular valvuloseptal morphogenesis. Circ Res. 1995;77(1):1-6.

3. Soor GS, et al. Hypertrophic cardiomyopathy: current understanding and treatment objectives. J Clin Pathol. 2009;62(3):226-235.

4. Strauss AW, Johnson MC. The genetic basis of pediatric cardiovascular disease. Semin Perinatol. 1996;20(6):564-576.

5. Maron BJ. Hypertrophic cardiomyopathy in childhood. Pediatr Clin North Am. 2004;51(5):1305-1346.

6. Anan R, et al. Prognostic implications of novel beta cardiac myosin heavy chain gene mutations that cause familial hypertrophic cardiomyopathy. J Clin Invest. 1994;93(1):280-285.

7. Marian AJ, Roberts R. Recent advances in the molecular genetics of hypertrophic cardiomyopathy. Circulation. 1995;92(5):1336-1347.

8. Schwartz K, Carrier L, Guicheney P, Komajda M. Molecular basis of familial cardiomyopathies. Circulation. 1995;91(2):532-540.

9. Niimura $\mathrm{H}$, et al. Mutations in the gene for cardiac myosin-binding protein $\mathrm{C}$ and late-onset familial hypertrophic cardiomyopathy. $N$ Engl J Med. 1998;338(18):1248-1257.

10. Clerk A, et al. Signaling pathways mediating cardiac myocyte gene expression in physiological and stress responses. J Cell Physiol. 2007;212(2):311-322.

11. Feng G. SHP-2 Tyrosine Phosphatase: Signaling one cell or many. Experimental Cell Research. 1999;253(1):47-54.

12. Tonks NK, Neel BG. Combinatorial control of the specificity of protein tyrosine phosphatases. Curr Opin Cell Biol. 2001;13(2):182-195.

13. Hof P, Pluskey S, Dhe-Pagganon S, Eck MJ, Shoelson SE. Crystal structure of the tyrosine phosphatase SHP-2. Cell. 1998;92(4):441-450.

14. Barford D, Neel BG. Revealing mechanisms for SH2 domain mediated regulation of the protein tyrosine phosphatase SHP-2. Structure. 1998;6(3):249-254.

15. O'Reilly AM, Neel BG. Structural seterminants of SHP-2 function and specificity in Xenopus mesoderm induction. Mol Cell Biol. 1998;18(1):161-177.

16. Kontaridis MI, et al. Deletion of Ptpn11 (Shp2) in cardiomyocytes causes dilated cardiomyopathy via effects on the extracellular signal-regulated kinase/ mitogen-activated protein kinase and RhoA signaling pathways. Circulation. 2008;117(11):1423-1435.

17. Feng G-S, Hui C-C, Pawson T. SH2-containing phosphotyrosine phosphatase as a target of protein-tyrosine kinases. Science. 1993;259(5101):1607-1611.

18. Neel BG, Gu H, Pao L. The 'Shp'ing news: SH2 domain-containing tyrosine phosphatases in cell signaling. Trends Biochem Sci. 2003;28(6):284-293.

19. Tartaglia M, Gelb BD. Noonan syndrome and related disorders: genetics and pathogenesis. Annu Rev Genomics Hum Genet. 2005; 6:45-68.

20. Gorlin RJ, Anderson RC, Moller JH. The Leopard (multiple lentigines) syndrome revisited. Birth Defects Orig Artic Ser. 1971;07(4):110-115.

21. Tartaglia M, et al. Mutations in PTPN11, encoding the protein tyrosine phosphatase SHP-2, cause Noonan syndrome. Nat Genet. 2001;29(4):465-468.

22. Roberts AE, et al. Germline gain-of-function mutations in SOS1 cause Noonan syndrome. Nat Genet. 2007;39(1):70-74.

23. Tartaglia M, et al. Gain-of-function SOS1 mutations cause a distinctive form of Noonan syndrome.
Nat Genet. 2007;39(1):75-79.

24. Schubbert $S$, et al. Germline KRAS mutations cause Noonan syndrome. Nat Genet. 2006;38(3):331-336.

25. Cirstea IC, et al. A restricted spectrum of NRAS mutations causes Noonan syndrome. Nat Genet. 2010;42(1):27-29.

26. Cordeddu V, et al. Mutation of SHOC2 promotes aberrant protein $\mathrm{N}$-myristoylation and causes Noonan-like syndrome with loose anagen hair. Nat Genet. 2009;41(9):1022-1026.

27. Pandit B, et al. Gain-of-function RAF1 mutations cause Noonan and LEOPARD syndromes with hypertrophic cardiomyopathy. Nat Genet. 2007;39(8):1007-1012.

28. Razzaque MA, et al. Germline gain-of-function mutations in RAF1 cause Noonan syndrome. Nat Genet. 2007;39(8):1013-1017.

29. Tidyman WE, Rauen KA. Noonan, Costello and cardio-facio-cutaneous syndromes: dysregulation of the Ras-MAPK pathway. Expert Rev Mol Med. 2008; 10:e37.

30. Rauen KA. HRAS and the Costello syndrome. Clin Genet. 2007;71(2):101-108.

31. Tartaglia M, et al. PTPN11 mutations in Noonan syndrome: molecular spectrum, genotype-phenotype correlation, and phenotypic heterogeneity. Am J Hum Genet. 2002;70(6):1555-1563.

32. Burch M, Sharland M, Shinebourne E, Smith G, Patton M, McKenna W. Cardiologic abnormalities in Noonan syndrome: phenotypic diagnosis and echocardiographic assessment of 118 patients. J Am Coll Cardiol. 1993;22(4):1189-1192.

33. Marino B, Digilio MC, Toscano A, Giannotti A, Dallapiccola B. Congenital heart diseases in children with Noonan syndrome: An expanded cardiac spectrum with high prevalence of atrioventricular canal. J Pediatr. 1999;135(6):703-706.

34. Ogata T, Yoshida R. PTPN11 mutations and genotype-phenotype correlations in Noonan and LEOPARD syndromes. Pediatr Endocrinol Rev. 2005 2(4):669-674

35. Sarkozy A, et al. Correlation between PTPN11 gene mutations and congenital heart defects in Noonan and LEOPARD syndromes. J Med Genet. 2003;40(9):704-708.

36. Sarkozy A, Digilio MC, Dallapiccola B. Leopard syndrome. Orphanet J Rare Dis. 2008;3:13.

37. Limongelli G, Hawkes L, Calabro R, McKenna WJ, Syrris P. Mutation screening of the PTPN11 gene in hypertrophic cardiomyopathy. Eur J Med Genet. 2006;49(5):426-430.

38. Digilio MC, et al. LEOPARD syndrome: Clinical diagnosis in the first year of life. Am J Med Genet A. 2006;140(7):740-746.

39. Kontaridis MI, Swanson KD, David FS, Barford D, Neel BG. PTPN11 (Shp2) mutations in LEOPARD syndrome have dominant negative, not activating, effects. J Biol Chem. 2006;281(10):6785-6792.

40. Tartaglia $\mathrm{M}$, et al. Diversity and functional consequences of germline and somatic PTPN11 mutations in human disease. Am J Hum Genet. 2006; 78(2):279-290.

41. Hanna N, et al. Reduced phosphatase activity of SHP-2 in LEOPARD syndrome: consequences for PI3K binding on Gab1. FEBS Lett. 2006 ; 580(10):2477-2482

42. Fragale A, Tartaglia M, Wu J, Gelb BD. Noonan syndrome-associated SHP2/PTPN11 mutants cause EGF-dependent prolonged GAB1 binding and sustained ERK2/MAPK1 activation. Hum Mutat. 2004;23(3):267-277.
43. Araki T, et al. Mouse model of Noonan syndrome reveals cell type- and gene dosage-dependent effects of Ptpn11 mutation. Nat Med. 2004;10(8):849-857.

44. Keilhack H, David FS, McGregor M, Cantley LC, Neel BG. Diverse biochemical properties of Shp2 mutants. Implications for disease phenotypes. J Biol Chem. 2005;280(35):30984-30993.

45. Niihori T, et al. Functional analysis of PTPN11/ SHP-2 mutants identified in Noonan syndrome and childhood leukemia. J Hum Genet. 2005; 50(4):192-202.

46. Schubbert S, et al. Functional analysis of leukemiaassociated PTPN11 mutations in primary hematopoietic cells. Blood. 2005;106(1):311-317.

47. Oishi K, et al. Phosphatase-defective LEOPARD syndrome mutations in PTPN11 gene have gain-offunction effects during Drosophila development. Hum Mol Genet. 2009;18(1):193-201.

48. Jopling C, van Geemen D, den Hertog J. Shp2 knockdown and Noonan/LEOPARD mutant Shp2induced gastrulation defects. PLoS Genet. 2007; 3(12): e225.

49. Stewart RA, et al. Phosphatase-dependent and -independent functions of Shp2 in neural crest cells underlie LEOPARD syndrome pathogenesis. Dev Cell. 2010;18(5):750-762.

50. Legius E, Schrander-Stumpel C, Schollen E, PullesHeintzberger C, Gewillig M, Fryns JP. PTPN11 mutations in LEOPARD syndrome. J Med Genet. 2002;39(8):571-574.

51. Liu P, Jenkins NA, Copeland NG. A highly efficient recombineering-based method for generating conditional knockout mutations. Genome Res. 2003;13(3):476-484.

52. Loh ML, et al. Mutations in PTPN11 implicate the SHP-2 phosphatase in leukemogenesis. Blood. 2004;103(6):2325-2331.

53. Tartaglia $\mathrm{M}$, et al. Genetic evidence for lineagerelated and differentiation stage-related contribution of somatic PTPN11 mutations to leukemogenesis in childhood acute leukemia. Blood. 2004;104(2):307-313

54. Chan G, Kalaitzidis D, Neel BG. The tyrosine phosphatase Shp2 (PTPN11) in cancer. Cancer Metastasis Rev. 2008;27(2):179-192.

55. Tartaglia M, Gelb BD. Noonan syndrome and related disorders: genetics and pathogenesis. Annu Rev Genomics Hum Genet. 2005;6:45-68.

56. Digilio MC, Capolino R, Marino B, Sarkozy A, Dallapiccola B. Congenital intrahepatic portosystemic venous shunt: an unusual feature in LEOPARD syndrome and in neurofibromatosis type $1 . A m J$ Med Genet A. 2005;134(4):457-458.

57. Digilio MC, et al. Grouping of multiple-lentigines/ LEOPARD and Noonan syndromes on the PTPN11 gene. Am J Hum Genet. 2002;71(2):389-394.

58. Mihl C, Dassen WR, Kuipers H. Cardiac remodelling: concentric versus eccentric hypertrophy in strength and endurance athletes. Neth Heart J. 2008;16(4):129-133.

59. Tartaglia M, Zampino G, Gelb BD. Noonan syndrome: clinical aspects and molecular pathogenesis. Mol Syndromol. 2010;1(1):2-26.

60. Zenker M. Genetic and pathogenetic aspects of Noonan syndrome and related disorders. Horm Res. 2009;72 suppl 2:57-63.

61. Schoenfeld JR, et al. Distinct molecular phenotypes in murine cardiac muscle development, growth, and hypertrophy. J Mol Cell Cardiol. 1998; 30(11):2269-2280.

62. Nakagawa $\mathrm{O}$, et al. Rapid transcriptional activa- 
tion and early mRNA turnover of brain natriuretic peptide in cardiocyte hypertrophy. Evidence for brain natriuretic peptide as an "emergency" cardiac hormone against ventricular overload. J Clin Invest. 1995;96(3):1280-1287.

63. Wu X, et al. MEK-ERK pathway modulation ameliorates disease phenotypes in a mouse model of Noonan syndrome associated with the Raf1L613V mutation. J Clin Invest. 2011:121(3):1009-1025.

64. Hakak Y, Hsu YS, Martin GS. Shp-2 mediates v-Srcinduced morphological changes and activation of the anti-apoptotic protein kinase Akt. Oncogene. 2000;19(28):3164-3171.

65. Wu CJ, O'Rourke DM, Feng GS, Johnson GR, Wang Q, Greene MI. The tyrosine phosphatase SHP-2 is required for mediating phosphatidylinositol 3kinase/Akt activation by growth factors. Oncogene. 2001;20(42):6018-6025.

66. Zhang SQ, et al. Receptor-specific regulation of phosphatidylinositol 3'-kinase activation by the protein tyrosine phosphatase Shp2. Mol Cell Biol. 2002;22(12):4062-4072.

67. Marin TM, et al. Shp2 negatively regulates growth in cardiomyocytes by controlling focal adhesion kinase/Src and mTOR pathways. Circ Res. 2008; 103(8):813-824.

68. Masoumi A, Reed-Gitomer B, Kelleher C, Schrier RW. Potential pharmacological interventions in polycystic kidney disease. Drugs. 2007;67(17):2495-2510.

69. Kim DH, Sabatini DM. Raptor and mTOR: subunits of a nutrient-sensitive complex. Curr Top Microbiol Immunol. 2004;279:259-270.

70. Chen XG, et al. Rapamycin regulates Akt and ERK phosphorylation through mTORC1 and mTORC2 signaling pathways. Mol Carcinog. 2010; 49(6):603-610

71. Shillingford JM, Piontek KB, Germino GG, Weimbs T. Rapamycin ameliorates PKD resulting from conditional inactivation of Pkd1. J Am Soc Nephrol. 2010;21(3):489-497.

72. Lee N, Woodrum CL, Nobil AM, Rauktys AE, Messina MP, Dabora SL. Rapamycin weekly maintenance dosing and the potential efficacy of combination sorafenib plus rapamycin but not atorvastatin or doxycycline in tuberous sclerosis preclinical models. BMC Pharmacol. 2009;9:8.

73. Edouard T, et al. Functional effects of PTPN11 (SHP2) mutations causing LEOPARD syndrome on epidermal growth factor-induced phosphoinositide 3-kinase/AKT/glycogen synthase kinase 3beta signaling. Mol Cell Biol. 2010;30(10):2498-2507.

74. Wang Y, Su B, Sah VP, Brown JH, Han J, Chien KR. Cardiac hypertrophy induced by mitogen-activated protein kinase kinase 7, a specific activator for c-Jun $\mathrm{NH} 2$-terminal kinase in ventricular muscle cells. J Biol Chem. 1998;273(10):5423-5426.

75. Ng DC, Court NW, dos Remedios CG, Bogoyevitch MA. Activation of signal transducer and activator of transcription (STAT) pathways in failing human hearts. Cardiovasc Res. 2003;57(2):333-346.

76. Krenz M, Gulick J, Osinska HE, Colbert MC Molkentin JD, Robbins J. Role of ERK1/2 signaling in congenital valve malformations in Noonan syndrome. Proc Natl Acad Sci U S A. 2008; 105(48):18930-18935.

77. Araki T, Chan G, Newbigging S, Morikawa L, Bronson RT, Neel BG. Noonan syndrome cardiac defects are caused by PTPN11 acting in endocardium to enhance endocardial-mesenchymal transformation. Proc Natl Acad Sci U S A. 2009; 106(12):4736-4741.
78. Allanson JE, et al. The face of Noonan syndrome: Does phenotype predict genotype. Am JMed Genet A. 2010;152A(8):1960-1966.

79. Chen PC, et al. Activation of multiple signaling pathways causes developmental defects in mice with a Noonan syndrome-associated Sos 1 mutation. J Clin Invest. 2010;120(12):4353-4365.

80. Yoshida R, Nagai T, Hasegawa T, Kinoshita E, Tanaka T, Ogata T. Two novel and one recurrent PTPN11 mutations in LEOPARD syndrome. Am J Med Genet A. 2004;130A(4):432-434.

81. Nishikawa T, Ishiyama S, Shimojo T, Takeda K, Kasajima T, Momma K. Hypertrophic cardiomyopathy in Noonan syndrome. Acta Paediatr Jpn. 1996;38(1):91-98.

82. Digilio MC, et al. Noonan syndrome and aortic coarctation. Am J Med Genet. 1998;80(2):160-162.

83. Digilio MC, et al. LEOPARD syndrome: clinical diagnosis in the first year of life. Am J Med Genet A. 2006;140(7):740-746.

84. Dorn GW 2nd, Force T. Protein kinase cascades in the regulation of cardiac hypertrophy. J Clin Invest. 2005;115(3):527-537.

85. Carvajal-Vergara X, et al. Patient-specific induced pluripotent stem-cell-derived models of LEOPARD syndrome. Nature. 2010;465(7299):808-812.

86 . Boluyt MO, et al. Rapamycin inhibits alpha 1adrenergic receptor-stimulated cardiac myocyte hypertrophy but not activation of hypertrophyassociated genes. Evidence for involvement of p70 S6 kinase. Circ Res. 1997;81(2):176-186.

87. Wang L, Proud CG. Ras/Erk signaling is essential for activation of protein synthesis by Gq proteincoupled receptor agonists in adult cardiomyocytes. Circ Res. 2002;91(9):821-829.

88. Sadoshima J, Izumo S. Rapamycin selectively inhibits angiotensin II-induced increase in protein synthesis in cardiac myocytes in vitro. Potential role of 70-kD S6 kinase in angiotensin II-induced cardiac hypertrophy. Circ Res. 1995;77(6):1040-1052.

89. Wang L, Wang X, Proud CG. Activation of mRNA translation in rat cardiac myocytes by insulin involves multiple rapamycin-sensitive steps. Am J Physiol Heart Circ Physiol. 2000;278(4):H1056-H1068.

90. Shioi T, et al. Akt/protein kinase B promotes organ growth in transgenic mice. Mol Cell Biol. 2002;22(8):2799-2809.

91. Shioi T, et al. Rapamycin attenuates loadinduced cardiac hypertrophy in mice. Circulation. 2003;107(12):1664-1670.

92. Shen WH, et al. Cardiac restricted overexpression of kinase-dead mammalian target of rapamycin (mTOR) mutant impairs the mTOR-mediated signaling and cardiac function. J Biol Chem. 2008; 283(20):13842-13849.

93. Takeda $\mathrm{N}$, et al. Cardiac fibroblasts are essential for the adaptive response of the murine heart to pressure overload. J Clin Invest. 2010;120(1):254-265.

94. Thum T, et al. MicroRNA-21 contributes to myocardial disease by stimulating MAP kinase signalling in fibroblasts. Nature. 2008;456(7224):980-984.

95 . Ieda $\mathrm{M}$, et al. Cardiac fibroblasts regulate myocardial proliferation through beta1 integrin signaling. Dev Cell. 2009;16(2):233-244.

96. Miragoli M, Gaudesius G, Rohr S. Electrotonic modulation of cardiac impulse conduction by myofibroblasts. Circ Res. 2006;98(6):801-810.

97. Agah R, Frenkel PA, French BA, Michael LH, Overbeek PA, Schneider MD. Gene recombination in postmitotic cells. Targeted expression of Cre recombinase provokes cardiac-restricted, site-specific rearrangement in adult ventricular muscle in vivo. J Clin Invest. 1997;100(1):169-179.

98. Alexandre J, Raymond E, Armand JP. [Rapamycin and CCI-779]. Bull Cancer. 1999;86(10):808-811.

99. Soesanto W, et al. Mammalian target of rapamycin is a critical regulator of cardiac hypertrophy in spontaneously hypertensive rats. Hypertension. 2009;54(6):1321-1327.

100.Gao XM, et al. Inhibition of mTOR reduces chronic pressure-overload cardiac hypertrophy and fibrosis. JHypertens. 2006;24(8):1663-1670.

101.Lieberthal W, Levine JS. The role of the mammalian target of rapamycin (mTOR) in renal disease. $J \mathrm{Am}$ Soc Nephrol. 2009;20(12):2493-2502.

102. Rosner M, Hanneder M, Siegel N, Valli A, Fuchs $\mathrm{C}$, Hengstschlager M. The mTOR pathway and its role in human genetic diseases. Mutat Res. 2008; 659(3):284-292

103. Serra AL, et al. Clinical proof-of-concept trial to assess the therapeutic effect of sirolimus in patients with autosomal dominant polycystic kidney disease: SUISSE ADPKD study. BMC Nephrol. 2007;8:13.

104. Wuthrich RP, Serra AL. Mammalian target of rapamycin and autosomal dominant polycystic kidney disease. Transplant Proc. 2009;41(6 suppl):S18-S20.

105.Dancey JE, Curiel R, Purvis J. Evaluating temsirolimus activity in multiple tumors: a review of clinical trials. Semin Oncol. 2009;36 suppl 3:S46-S58.

106. Mattoon DR, Lamothe B, Lax I, Schlessinger J. The docking protein Gab1 is the primary mediator of EGF-stimulated activation of the PI-3K/Akt cell survival pathway. BMC Biol. 2004;2:24.

107. Matsuo K, et al. Altered glucose homeostasis in mice with liver-specific deletion of Src homology phosphatase 2. J Biol Chem. 2010;285(51):39750-39758.

108.Lupu F, Terwilliger JD, Lee K, Segre GV, Efstratiadis A. Roles of growth hormone and insulin-like growth factor 1 in mouse postnatal growth. Dev Biol. 2001;229(1):141-162.

109. Shioi T, et al. The conserved phosphoinositide 3-kinase pathway determines heart size in mice. EMBO J. 2000;19(11):2537-2548.

110.McMullen JR, et al. Phosphoinositide 3-kinase (p110alpha) plays a critical role for the induction of physiological, but not pathological, cardiac hypertrophy. Proc Natl Acad Sci U S A. 2003; 100(21):12355-12360

111. Crackower MA, et al. Regulation of myocardial contractility and cell size by distinct PI3K-PTEN signaling pathways. Cell. 2002;110(6):737-749.

112. Patrucco E, et al. PI3Kgamma modulates the cardiac response to chronic pressure overload by distinct kinase-dependent and -independent effects. Cell. 2004;118(3):375-387.

113.Fujio Y, Nguyen T, Wencker D, Kitsis RN, Walsh K. Akt promotes survival of cardiomyocytes in vitro and protects against ischemia-reperfusion injury in mouse heart. Circulation. 2000;101(6):660-667.

114. Schiekofer S, Shiojima I, Sato K, Galasso G, Oshima Y, Walsh K. Microarray analysis of Akt1 activation in transgenic mouse hearts reveals transcript expression profiles associated with compensatory hypertrophy and failure. Physiol Genomics. 2006; 27(2):156-170.

115.Liao R, et al. Cardiac-specific overexpression of GLUT1 prevents the development of heart failure attributable to pressure overload in mice. Circulation. 2002;106(16):2125-2131.

116.Zhu X, et al. Three-dimensional structures of acidic and basic fibroblast growth factors. Science. 1991;251(4989):90-93. 